Durán-González, A., Laguarda-Figueras, A., Solís-Marín,

F.A., Gracia, A. \& Vázquez-Bader, A.R. (2021).

Biodiversidad de ofiuroideos (Echinodermata:

Ophiuroidea) del talud de la Península de Yucatán,

México. Revista de Biología Tropical, 69(S1), 132-

153. DOI 10.15517/rbt.v69iSuppl.1.46337

DOI 10.15517/rbt.v69iSuppl.1.46337

\title{
Biodiversidad de ofiuroideos (Echinodermata: Ophiuroidea) del talud de la Península de Yucatán, México
}

\author{
Alicia Durán-González ${ }^{1}$ \\ Alfredo Laguarda-Figueras ${ }^{1}$ \\ Francisco Alonso Solís-Marín ${ }^{1}$ \\ Adolfo Gracia ${ }^{2}$ \\ Ana Rosa Vázquez-Bader ${ }^{2}$
}

1. Laboratorio de Sistemática y Ecología de Equinodermos, Instituto de Ciencias del Mar y Limnología, Universidad Nacional Autónoma de México, Ciudad de México, México; aliciad@cmarl.unam.mx, laguarda@cmarl.unam.mx, fasolis@cmarl.unam.mx

2. Laboratorio Ecología Pesquera de Crustáceos, Instituto de Ciencias del Mar y Limnología, Universidad Nacional Autónoma de México, Ciudad de México, México; gracia@unam.mx, ana-rosav@hotmail.com

$$
\text { Recibido 21-VI-2020. Corregido 11-IX-2020. Aceptado 20-IX-2020. }
$$

\section{ABSTRACT \\ Biodiversity of ophiuroids (Echinodermata: Ophiuroidea) from the slope of the Yucatan Peninsula, Mexico}

\begin{abstract}
Introduction: The Ophiuroidea class is the most diverse of the Phylum Echinodermata, with 2064 described species, which can be found from intertidal zones to abyssal depths, but the fauna of Yucatan peninsula needs more study. Objective: To describe the diversity of ophiuroids in the Yucatan continental slope. Methods: Information was compiled from four oceanographic surveys carried out in August 2005, June 2007 (BIOREPES 1 and 2: BRP1 and BRP2), April 2011 and August 2014 (COBERPES 2 and 6: CBP2 and CBP6). The specimens were obtained by trawling with a shrimp net in soft substrates and with a skimmer-type trawl dredge at a depth range of 200 to $1071 \mathrm{~m}$ off the states of Yucatán and Quintana Roo, Mexico. Results: We found members of two superorders, five orders, six suborders, five superfamilies, 18 families, 28 genera and 43 species. Conclusions: The northeast zone Yucatan Peninsula has at least 43 species, of which 19 are new records for the area.
\end{abstract}

Key words: taxonomy; new records; deep sea; Gulf of Mexico.

En la prospección de las zonas costeras y mares de la República Mexicana efectuada por el ICML-UNAM con el B/O "Justo Sierra", a partir del año 2000 las investigaciones se han abocado al conocimiento de la biodiversidad del mar profundo incrementando el conocimiento de sus recursos naturales y biodiversidad. En el Golfo de México se considera la existencia de 512 especies de equinodermos, la cual aproximadamente corresponde al 7\% de la fauna de equinodermos mundial (Pawson, Vance, Messing, Solís-Marín \& Mah, 2009). La clase Ophiuroidea es la más diversa del Phylum Echinodermata con 2064 especies descritas (Stöhr, O'Hara, \& Thuy, 2012), que se pueden encontrar desde las zonas intermareales hasta profundidades abisales (Pawson, 2007). A nivel mundial la diversidad del grupo 
ha sido valorada por Stöhr et al., (2012) con base en la compilación efectuada en la base de datos WoRMS (World Register of Marine Species) donde se consideran 2064 especies descritas, de las cuales 1412 se distribuyen en mar profundo. Así mismo, como componente fundamental de los fondos marinos, dadas sus características biológicas y amplia distribución han adquirido importancia como sustento de grandes bases de datos, los cuales han permitido desarrollar modelos predictivos a fin de encontrar patrones de diversidad del mar profundo (Woolley, Tittensor, Dunstan, Guillera-Arroita, Lahoz-Monfort, Wintle, Worm \& O'Hara, 2016).

Entre los trabajos que presentan información taxonómica sobre equinodermos para el Golfo de México se deben mencionar a DuránGonzález, Laguarda-Figueras, Solís-Marín, Buitrón-Sánchez, Gust-Ahearn y Torres-Vega (2005) donde se presenta un listado de 209 especies, de las cuales 49 especies correspondientes a la clase Ophiuroidea; particularmente para la clase Ophiuroidea se tiene el reporte de 68 especies en Hernández-Herrejón, Solís-Marín y Laguarda-Figueras (2008) y 98 especies en Laguarda-Figueras, Hernández-Herrejón, Solís-Marín y Durán-González (2009). El estado de conocimiento de los ofiuroideos se ha incrementado a través de los años, sin embargo, son escasos las especies referidas a ambientes profundos ( $>200 \mathrm{~m}$ ) del Golfo de México y que se encuentren depositados en una Colección mexicana por la dificultad de obtenerlas. Considerando al Golfo de México como el área de mayor riqueza de los mares territoriales de México, para la clase Ofiuroidea Solís-Marín, Laguarda-Figueras y Honey-Escandón (2014) reportan 79 especies, correspondiendo 46 especies para el estado de Yucatán. Así mismo por su importancia Felder, Camp y Tunnell (2009) elaboraron el estado de conocimiento del Golfo de México donde 512 especies representan al grupo Echinodermata.

Con el fin de ampliar el conocimiento de la biodiversidad presente en el Noreste de la Península de Yucatán y obtener un inventario de especies de la clase Ophiuroidea del Talud de los estados de Yucatán y Quintana Roo, se analizaron los organismos resguardados en la Colección Nacional de Equinodermos (CNE) del Instituto de Ciencias del Mar y Limnología (ICML) de la Universidad Nacional Autónoma de México (UNAM) obtenidos en cuatro cruceros oceanográficos, enfatizando en las nuevas especies que se han obtenido, ubicándolas en el nuevo contexto de la clasificación actual, en apoyo a los tomadores de decisiones para el cuidado y mantenimiento de los recursos naturales de México.

\section{MATERIALES Y MÉTODOS}

Para obtener la biodiversidad de equinodermos del noroeste de la Península de Yucatán se recopiló la información de cuatro campañas oceanográficas efectuadas en agosto 2005, junio 2007 en los cruceros BIOREPES 1 y 2 (Biodiversidad de Recursos Pesqueros Potenciales) BRP1 y BRP2, abril 2011 y agosto 2014, respectivamente y COBERPES 2 y 6: CBP2 y CBP6 (Comunidades Bentónicas y Recursos Pesqueros Potenciales del Mar Profundo del Golfo de México) integrando una red de 95 estaciones. Los ejemplares se obtuvieron mediante arrastres con red camaronera en sustratos suaves y con una draga de arrastre tipo Skimmer. La zona de estudio se encuentra entre las latitudes de $22^{\circ} 35^{\prime} 39^{\prime \prime}$ a $24^{\circ} 24^{\prime} 34^{\prime \prime}$ $\mathrm{N}$ y longitudes de $86^{\circ} 15^{\prime} 29^{\prime \prime}$ a $90^{\circ} 49^{\prime} 46^{\prime \prime} \mathrm{O}$ delimitando así el Talud, con un intervalo de profundidad de 200 a $1071 \mathrm{~m}$ frente a los estados de Yucatán y Quintana Roo, México (Fig. 1). Los ofiuroideos de la CNE se encuentran preservados en alcohol etílico al 70\% desde la recolecta. En el Tabla 1 se presentan los datos coorespondientes a 78 estaciones de muestreo donde hubo la presencia de ofiuroideos en la zona del talud: fecha, latitud $\mathrm{N}$, longitud $\mathrm{O}$ y profundidad, número de especies presentes en cada estación reconocidas con el número asignado en la clasificación.

Los ejemplares de la Clase Ophiuroidea, fueron determinados taxonómicamente mediante el empleo de claves y diagnosis de: Lyman (1869, 1871, 1875, 1878a, 1878b, 1882, 1883), 


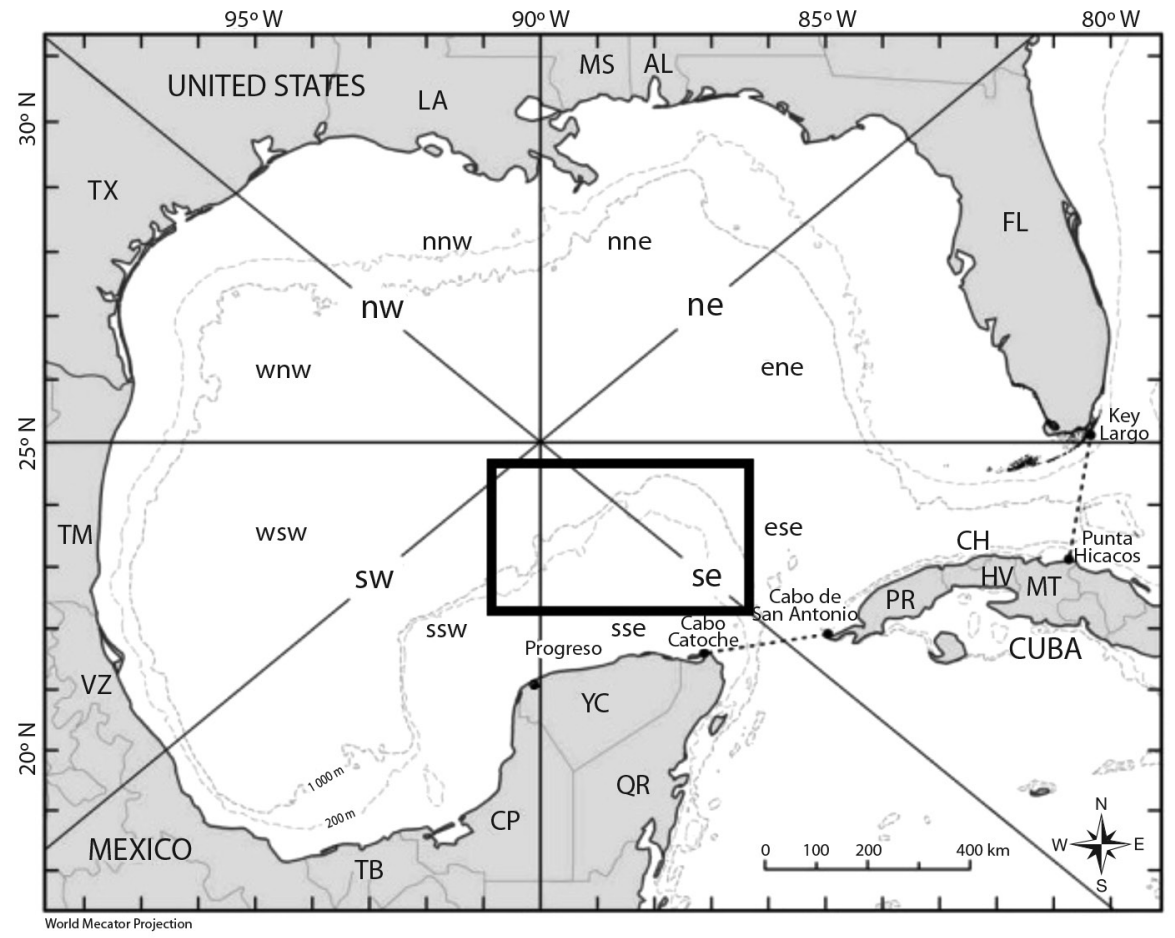

Fig. 1. Zona de estudio, Talud de la Península de Yucatán. Tomado de Felder, Camp \& Tunnell (2009).

Fig. 1. Study area, Slope of the Yucatan Peninsula. Takem from Felder, Camp \& Tunnell (2009).

Verrill (1899), A.H. Clark (1936), Cherbonnier (1959), Madsen (1977), y Paterson (1985), las especies se ubicaron en el contexto de la clasificación propuesta por O'Hara, Hugall, Thuy, Stöhr, y Martynov (2017) y O’Hara, Stöhr, Hugall, Thuy, y Martynov (2018). Todos los ejemplares se encuentran depositados en la CNE "Dra. María Elena Caso M." del ICML, UNAM. Se presentan nuevos registros con fotografías (región oral y aboral) tomadas con un microscopio Multifocal Leica Z16APOA. KL1500, representados con un asterisco previo al nombre de la especie en la clasificación; las referencias de identificación; rango de distribución batimétrica obtenida, material examinado con el número de ejemplares revisados, seguidos por el número de catálogo de la CNE (ICML-UNAM) y número de estación.

\section{RESULTADOS}

El inventario está integrado con el seguimiento de 2755 ejemplares de ofiuroideos obtenidos en 78 estaciones de muestreo, los cuales quedaron distribuidos en dos superórdenes, cinco órdenes, seis subórdenes, cinco superfamilias, 18 familias, 28 géneros y 43 especies. Se presenta la clasificación efectuada por O'Hara, Stöhr, Hugall, Thuy, y Martynov (2018). Las especies se enumeraron en este ordenamiento para ubicarlas con el número asignado en la Tabla 1. 
TABLA 1

Estaciones de muestreo para las especies de ofiuroideos del Talud Península de Yucatán, obtenidas en las Campañas Oceanográficas BIOREPES 1 y 2 (BRP1 y BRP2) y COBERPES 2 y 6 (CP2 y CP6)

TABLE 1

Sampling stations for ophiuroid species of the Yucatan Peninsula Slope, obtained in the Oceanographic Campaigns BIOREPES 1 y 2 (BRP1 and BRP2) and COBERPES 2 and 6 (CP2 and CP6)

\begin{tabular}{|c|c|c|c|c|c|c|c|}
\hline Estación & Campaña & Fecha & Latitud N & Longitud $\mathrm{O}$ & $\begin{array}{l}\text { Profundidad } \\
\text { (m) }\end{array}$ & $\begin{array}{c}\text { Total } \\
\text { Especies }\end{array}$ & $\begin{array}{l}\mathrm{N}^{0} \text { de especie } \\
\text { registrada }\end{array}$ \\
\hline E1 & BRP1.5 & Agosto 8, 2005 & $23^{\circ} 23^{\prime} 16^{\prime \prime}$ & $89^{\circ} 46^{\prime} 36^{\prime \prime}$ & 257 & 1 & 37 \\
\hline E2 & BRP1.7 & Agosto 9, 2005 & $23^{\circ} 29^{\prime} 19^{\prime \prime}$ & $89^{\circ} 46^{\prime} 51^{\prime \prime}$ & 359 & 2 & 1,17 \\
\hline E3 & BRP1.12 & Agosto 9, 2005 & $23^{\circ} 31^{\prime} 26^{\prime \prime}$ & $89^{\circ} 46^{\prime} 38^{\prime \prime}$ & 394 & 2 & 15,16 \\
\hline E5 & BRP1.18 & Agosto 10, 2005 & $23^{\circ} 20^{\prime} 40^{\prime \prime}$ & $89^{\circ} 48^{\prime} 30^{\prime \prime}$ & 246 & 3 & $10,14,16$ \\
\hline E6 & BRP1.19 & Agosto 10, 2005 & $23^{\circ} 20^{\prime} 19^{\prime \prime}$ & $89^{\circ} 49^{\prime} 03^{\prime \prime}$ & 245 & 3 & $10,11,14$ \\
\hline E7 & BRP1.20 & Agosto 10, 2005 & $23^{\circ} 24^{\prime} 20^{\prime \prime}$ & $89^{\circ} 49^{\prime} 52^{\prime \prime}$ & 315 & 3 & $10,14,41$ \\
\hline E8 & BRP1.22 & Agosto 10, 2005 & $23^{\circ} 30^{\prime} 45^{\prime \prime}$ & $89^{\circ} 49^{\prime} 57^{\prime \prime}$ & 427 & 6 & $7,10,11,14,16,40$ \\
\hline E9 & BRP1.26 & Agosto 11, 2005 & $24^{\circ} 02^{\prime} 29^{\prime \prime}$ & $88^{\circ} 11^{\prime} 58^{\prime \prime}$ & 311 & 6 & $14,17,35,38,40,41$ \\
\hline E10 & BRP1.27 & Agosto 11, 2005 & $24^{\circ} 08^{\prime} 14^{\prime \prime}$ & $88^{\circ} 12^{\prime} 18^{\prime \prime}$ & 352 & 1 & 42 \\
\hline E11 & BRP1.31 & Agosto 11, 2005 & $24^{\circ} 16^{\prime} 36^{\prime \prime}$ & $88^{\circ} 12^{\prime} 34^{\prime \prime}$ & 455 & 3 & $9,11,23$ \\
\hline E12 & BRP1.35 & Agosto 12, 2005 & $23^{\circ} 49^{\prime} 26^{\prime \prime}$ & $88^{\circ} 13^{\prime} 18^{\prime \prime}$ & 215 & 1 & 4 \\
\hline E13 & BRP1.36 & Agosto 12, 2005 & $23^{\circ} 49^{\prime} 17^{\prime \prime}$ & $88^{\circ} 13^{\prime} 23^{\prime \prime}$ & 214 & 2 & 10,11 \\
\hline E14 & BRP1.42 & Agosto 13, 2005 & $23^{\circ} 46^{\prime} 12^{\prime \prime}$ & $87^{\circ} 06^{\prime} 38^{\prime \prime}$ & 618 & 4 & $7,9,12,19$ \\
\hline E15 & BRP1.47 & Agosto 13, 2005 & $23^{\circ} 48^{\prime} 22^{\prime \prime}$ & $87^{\circ} 08^{\prime} 09^{\prime \prime}$ & 613 & 7 & $2,6,7,8,9,10,12$ \\
\hline E16 & BRP1.48 & Agosto 13, 2005 & $23^{\circ} 52^{\prime} 33^{\prime \prime}$ & $87^{\circ} 05^{\prime} 51^{\prime \prime}$ & 700 & 4 & $9,12,24,26$ \\
\hline E17 & BRP1.50 & Agosto 14, 2005 & $23^{\circ} 52^{\prime} 57^{\prime \prime}$ & $87^{\circ} 10^{\prime} 47^{\prime \prime}$ & 620 & 4 & $5,7,12,18$ \\
\hline E18 & BRP1.55 & Agosto 14, 2005 & $23^{\circ} 55^{\prime} 30^{\prime \prime}$ & $87^{\circ} 11^{\prime} 18^{\prime \prime}$ & 645 & 4 & $7,11,12,36$ \\
\hline E20 & BRP2.E6 & Junio 1, 2007. & $23^{\circ} 24^{\prime} 14^{\prime \prime}$ & $90^{\circ} 01^{\prime} 39^{\prime \prime}$ & 681 & 1 & 22 \\
\hline E21 & BRP2.E7 & Junio 1, 2007. & $23^{\circ} 32^{\prime} 38^{\prime \prime}$ & $89^{\circ} 56^{\prime} 26^{\prime \prime}$ & 101 & 1 & 22 \\
\hline E22 & BRP2.E8 & Junio 1, 2007. & $23^{\circ} 33^{\prime} 13^{\prime \prime}$ & $89^{\circ} 56^{\prime} 19^{\prime \prime}$ & 1071 & 1 & 22 \\
\hline E23 & BRP2.E10 & Junio 1, 2007. & $23^{\circ} 10^{\prime} 50^{\prime \prime}$ & $89^{\circ} 59^{\prime} 43^{\prime \prime}$ & 460 & 1 & 8 \\
\hline E24 & BRP2.E11 & Junio 2, 2007. & $23^{\circ} 14^{\prime} 57^{\prime \prime}$ & $89^{\circ} 59^{\prime} 32^{\prime \prime}$ & 536 & 2 & 8,9 \\
\hline E25 & BRP2.E12 & Junio 2, 2007. & $23^{\circ} 25^{\prime} 35^{\prime \prime}$ & $89^{\circ} 59^{\prime} 16^{\prime \prime}$ & 650 & 2 & 5,8 \\
\hline E27 & BRP2.E14 & Junio 2, 2007. & $23^{\circ} 27^{\prime} 34^{\prime \prime}$ & $90^{\circ} 05^{\prime} 30^{\prime \prime}$ & 863 & 1 & 6 \\
\hline E28 & BRP2.E16 & Junio 2, 2007. & $23^{\circ} 25^{\prime} 21^{\prime \prime}$ & $89^{\circ} 48^{\prime} 50^{\prime \prime}$ & 305 & 2 & 3,34 \\
\hline E29 & BRP2.E17 & Junio 2, 2007. & $23^{\circ} 23^{\prime} 36^{\prime \prime}$ & $89^{\circ} 46^{\prime} 24^{\prime \prime}$ & 254 & 2 & 3,34 \\
\hline E30 & CP2.A5 & Abril 8, 2011 & $23^{\circ} 34^{\prime} 42^{\prime \prime}$ & $89^{\circ} 34^{\prime} 04^{\prime \prime}$ & 351 & 2 & 3,34 \\
\hline E31 & CP2.A7 & Abril 9, 2011 & $23^{\circ} 30^{\prime} 58^{\prime \prime}$ & $89^{\circ} 49^{\prime} 25^{\prime \prime}$ & 422 & 4 & $5,21,23,24$ \\
\hline E32 & CP2.A8 & Abril 9, 2011 & $24^{\circ} 02^{\prime} 10^{\prime \prime}$ & $88^{\circ} 10^{\prime} 36^{\prime \prime}$ & 308 & 5 & $18,20,34,35,42$ \\
\hline E33 & CP2.A10 & Abril 10, 2011 & $24^{\circ} 23^{\prime} 14^{\prime \prime}$ & $87^{\circ} 44^{\prime} 00^{\prime \prime}$ & 749 & 1 & 25 \\
\hline E34 & CP2.A11 & Abril 10, 2011 & $24^{\circ} 22^{\prime} 07^{\prime \prime}$ & $87^{\circ} 42^{\prime} 11^{\prime \prime}$ & 746 & 1 & 1 \\
\hline E36 & CP2.A15 & Abril 11, 2011 & $24^{\circ} 24^{\prime} 34^{\prime \prime}$ & $87^{\circ} 38^{\prime} 13^{\prime \prime}$ & 820 & 3 & $25,28,29$ \\
\hline E37 & CP2.A16 & Abril 11, 2011 & $24^{\circ} 23^{\prime} 21^{\prime \prime}$ & $87^{\circ} 36^{\prime} 42^{\prime \prime}$ & 937 & 1 & 43 \\
\hline E38 & CP2.A17 & Abril 11, 2011 & $23^{\circ} 46^{\prime} 28^{\prime \prime}$ & $87^{\circ} 06^{\prime} 52^{\prime \prime}$ & 620 & 5 & $5,8,12,18,19$ \\
\hline E39 & CP2.A18 & Abril 11, 2011 & $23^{\circ} 56^{\prime} 05^{\prime \prime}$ & $87^{\circ} 04^{\prime} 11^{\prime \prime}$ & 772 & 2 & 26,28 \\
\hline E40 & CP2.A19 & Abril 11, 2011 & $23^{\circ} 38^{\prime} 29^{\prime \prime}$ & $87^{\circ} 04^{\prime} 50^{\prime \prime}$ & 572 & 3 & $1,8,12$ \\
\hline E41 & CP2.A20 & Abril 12, 2011 & $23^{\circ} 40^{\prime} 35^{\prime \prime}$ & $87^{\circ} 04^{\prime} 50^{\prime \prime}$ & 642 & 5 & $9,12,20,30,31$ \\
\hline $\mathrm{E} 42$ & CP2.A21 & Abril 12, 2011 & $23^{\circ} 23^{\prime} 19^{\prime \prime}$ & $86^{\circ} 53^{\prime} 04^{\prime \prime}$ & 595 & 5 & $5,12,19,28,36$ \\
\hline E43 & CP2.A22 & Abril 12, 2011 & $23^{\circ} 26^{\prime} 42^{\prime \prime}$ & $86^{\circ} 53^{\prime} 04^{\prime \prime}$ & 633 & 6 & $5,12,19,25,36,43$ \\
\hline E44 & CP2.A23 & Abril 12, 2011 & $23^{\circ} 31^{\prime} 13^{\prime \prime}$ & $86^{\circ} 50^{\prime} 10^{\prime \prime}$ & 735 & 3 & $5,12,31$ \\
\hline E45 & CP2.A24 & Abril 13, 2011 & $23^{\circ} 35^{\prime} 27^{\prime \prime}$ & $86^{\circ} 50^{\prime} 04^{\prime \prime}$ & 806 & 2 & 9,11 \\
\hline
\end{tabular}


TABLA 1 (Continuación) / TABLE 1 (Continued)

\begin{tabular}{|c|c|c|c|c|c|c|c|}
\hline Estación & Campaña & Fecha & Latitud N & Longitud $\mathrm{O}$ & $\begin{array}{l}\text { Profundidad } \\
\text { (m) }\end{array}$ & $\begin{array}{c}\text { Total } \\
\text { Especies }\end{array}$ & $\begin{array}{l}\mathrm{N}^{\circ} \text { de especie } \\
\text { registrada }\end{array}$ \\
\hline E46 & CP2.A25 & Abril 13, 2011 & $23^{\circ} 02^{\prime} 31^{\prime \prime}$ & $86^{\circ} 26^{\prime} 16^{\prime \prime}$ & 976 & 7 & $2,12,19,22,29,39,43$ \\
\hline E47 & CP2.A26 & Abril 13, 2011 & $23^{\circ} 00^{\prime} 12^{\prime \prime}$ & $86^{\circ} 31^{\prime} 33^{\prime \prime}$ & 814 & 5 & $13,25,28,29,32$ \\
\hline E48 & CP2.A28 & Abril 14, 2011 & $23^{\circ} 02^{\prime} 08^{\prime \prime}$ & $86^{\circ} 43^{\prime} 29^{\prime \prime}$ & 530 & 3 & $9,19,31$ \\
\hline E49 & CP2.A29 & Abril 14, 2011 & $22^{\circ} 48^{\prime} 35^{\prime \prime}$ & $86^{\circ} 25^{\prime} 20^{\prime \prime}$ & 865 & 3 & $5,29,43$ \\
\hline E50 & CP2.A30 & Abril 14, 2011 & $22^{\circ} 50^{\prime} 04^{\prime \prime}$ & $86^{\circ} 25^{\prime} 00^{\prime \prime}$ & 902 & 2 & 2,43 \\
\hline E51 & CP2.A31 & Abril 15, 2011 & $22^{\circ} 53^{\prime} 03^{\prime \prime}$ & $86^{\circ} 15^{\prime} 29^{\prime \prime}$ & 1040 & 6 & $2,5,12,18,29,43$ \\
\hline E52 & CP6. D1 & Agosto 11, 2014 & $23^{\circ} 38^{\prime} 58^{\prime \prime}$ & $87^{\circ} 04^{\prime} 52^{\prime \prime}$ & 580 & 5 & $5,15,19,23,34$ \\
\hline E53 & CP6. C5b & Agosto 11, 2014 & $23^{\circ} 23^{\prime} 31^{\prime \prime}$ & $86^{\circ} 53^{\prime} 12^{\prime \prime}$ & 598 & 1 & 12 \\
\hline E54 & CP6.C5r. & Agosto 12, 2014 & $23^{\circ} 23^{\prime} 33^{\prime \prime}$ & $86^{\circ} 53^{\prime} 14^{\prime \prime}$ & 597 & 7 & $5,8,12,19,28,36,43$ \\
\hline E55 & CP6. C6 & Agosto 12, 2014 & $23^{\circ} 26^{\prime} 23^{\prime \prime}$ & $86^{\circ} 52^{\prime} 46^{\prime \prime}$ & 638 & 2 & 12,19 \\
\hline E56 & CP6. D10 & Agosto 12, 2014 & $23^{\circ} 41^{\prime} 56^{\prime \prime}$ & $87^{\circ} 03^{\prime} 33^{\prime \prime}$ & 628 & 6 & $5,12,19,20,22,31$ \\
\hline E57 & CP6. D11 & Agosto 13, 2014 & $23^{\circ} 38^{\prime} 42^{\prime \prime}$ & $87^{\circ} 04^{\prime} 50^{\prime \prime}$ & 577 & 8 & $\begin{array}{c}5,9,12,19,22,28 \\
31,33\end{array}$ \\
\hline E58 & CP6. D12 & Agosto 13, 2014 & $23^{\circ} 40^{\prime} 28^{\prime \prime}$ & $87^{\circ} 01^{\prime} 20^{\prime \prime}$ & 645 & 5 & $5,8,19,36,43$ \\
\hline E59 & CP6. D13 & Agosto 13, 2014 & $23^{\circ} 45^{\prime} 28^{\prime \prime}$ & $87^{\circ} 06^{\prime} 11^{\prime \prime}$ & 619 & 5 & $5,12,19,36,43$ \\
\hline E60 & CP6. D18 & Agosto 14, 2014 & $24^{\circ} 22^{\prime} 00^{\prime \prime}$ & $87^{\circ} 47^{\prime} 03^{\prime \prime}$ & 615 & 3 & $5,12,19$ \\
\hline E61 & CP6. D19b & Agosto 14, 2014 & $24^{\circ} 21^{\prime} 12^{\prime \prime}$ & $87^{\circ} 46^{\prime} 40^{\prime \prime}$ & 587 & 1 & 12 \\
\hline E62 & CP6. E21 & Agosto 14, 2014 & $23^{\circ} 34^{\prime} 41^{\prime \prime}$ & $89^{\circ} 33^{\prime} 40^{\prime \prime}$ & 336 & 4 & $3,8,12,34$ \\
\hline E63 & CP6. E22 & Agosto 14, 2014 & $23^{\circ} 33^{\prime} 22^{\prime \prime}$ & $89^{\circ} 40^{\prime} 44^{\prime \prime}$ & 358 & 5 & $1,5,8,22,34$ \\
\hline E64 & CP6. E23 & Agosto 15, 2014 & $23^{\circ} 30^{\prime} 48^{\prime \prime}$ & $89^{\circ} 49^{\prime} 47^{\prime \prime}$ & 426 & 5 & $1,5,21,23,43$ \\
\hline E65 & CP6. E28 & Agosto 15, 2014 & $23^{\circ} 25^{\prime} 18^{\prime \prime}$ & $89^{\circ} 59^{\prime} 17^{\prime \prime}$ & 609 & 2 & 5,12 \\
\hline E66 & CP6. E29 & Agosto 15, 2014 & $23^{\circ} 22^{\prime} 27^{\prime \prime}$ & $89^{\circ} 59^{\prime} 17^{\prime \prime}$ & 582 & 7 & $5,8,12,19,22,28,43$ \\
\hline E67 & CP6. E30 & Agosto 15, 2014 & $23^{\circ} 24^{\prime} 54^{\prime \prime}$ & $89^{\circ} 59^{\prime} 19^{\prime \prime}$ & 606 & 4 & $5,12,22,23$ \\
\hline E68 & CP6. E31 & Agosto 16, 2014 & $23^{\circ} 15^{\prime} 45^{\prime \prime}$ & $89^{\circ} 59^{\prime} 31^{\prime \prime}$ & 547 & 7 & $5,8,12,19,22,27,31$ \\
\hline E69 & CP6. E34 & Agosto 16, 2014 & $23^{\circ} 13^{\prime} 44^{\prime \prime}$ & $89^{\circ} 58^{\prime} 13^{\prime \prime}$ & 392 & 2 & 8,34 \\
\hline E70 & CP6. E36 & Agosto 17, 2014 & $23^{\circ} 23^{\prime} 56^{\prime \prime}$ & $89^{\circ} 59^{\prime} 38^{\prime \prime}$ & 608 & 3 & $5,12,22$ \\
\hline E71 & CP6. E37 & Agosto 17, 2014 & $23^{\circ} 21^{\prime} 57^{\prime \prime}$ & $89^{\circ} 58^{\prime} 35^{\prime \prime}$ & 547 & 5 & $5,8,12,31,43$ \\
\hline E72 & CP6. F41 & Agosto 17, 2014 & $23^{\circ} 38^{\prime} 28^{\prime \prime}$ & $90^{\circ} 47^{\prime} 17^{\prime \prime}$ & 570 & 3 & $5,12,22$ \\
\hline E73 & CP6. F42 & Agosto 17, 2014 & $22^{\circ} 40^{\prime} 00^{\prime \prime}$ & $90^{\circ} 46^{\prime} 58^{\prime \prime}$ & 611 & 5 & $5,12,19,22,30$ \\
\hline E74 & CP6. F43 & Agosto 18, 2014 & $22^{\circ} 36^{\prime} 55^{\prime \prime}$ & $90^{\circ} 47^{\prime} 35^{\prime \prime}$ & 514 & 1 & 43 \\
\hline E75 & CP6. F44 & Agosto 18, 2014 & $22^{\circ} 35^{\prime} 39^{\prime \prime}$ & $90^{\circ} 49^{\prime} 46^{\prime \prime}$ & 477 & 2 & 8,43 \\
\hline E76 & CP6. F47 & Agosto 18, 2014 & $22^{\circ} 40^{\prime} 00^{\prime \prime}$ & $90^{\circ} 47^{\prime} 01^{\prime \prime}$ & 611 & 7 & $1,5,8,19,22,30,43$ \\
\hline E77 & CP6. F48 & Agosto 18, 2014 & $22^{\circ} 38^{\prime} 36^{\prime \prime}$ & $90^{\circ} 46^{\prime} 51^{\prime \prime}$ & 573 & 2 & 22,30 \\
\hline E78 & CP6. F49 & Agosto 18, 2014 & $22^{\circ} 37^{\prime} 41^{\prime \prime}$ & $90^{\circ} 46^{\prime} 29^{\prime \prime}$ & 540 & 4 & $12,22,20,43$ \\
\hline
\end{tabular}

Phylum Echinodermata Bruguière, 1791

Subphylum Asterozoa von Zittel, 1895

Clase Ophiuroidea Gray, 1840

Subclase Myophiuroidea Matsumoto, 1915

Infraclase Metophiurida Matsumoto, 1913

Superorden Euryophiurida O'Hara et al., 2017

I. Orden Euryalida Lamarck, 1816

Familia Asteronychidae Ljungman, 1867. 72 ejemplares en una especie.
1.- Asteronyx loveni Müller y Troschel, 1842

Referencias de identificación: Lyman 1883: 282, Lám. 8, Figs. 136-138; Müller \& Troschel 1842: 119, Lám. 10, Figs. 3-5. En Hernández-Herrejón et al. 2008: 93, Figs. 1 C, D y Laguarda-Figueras et al. 2009: 46, Lám. 5, se proporciona diagnosis, descripción y distribución geográfica y batimétrica.

Material examinado: 72 ejemplares. 65 ejemplares corresponden al área de Yucatán 
y siete a la de Quintana Roo, en un rango batimétrico obtenido de 359-746 m. Un ejemplar, ICML-UNAM 3.62.7, E2; 1 ejemplar, ICML-UNAM 9675, E34; 6 ejemplares, ICML-UNAM 9708, E40; 25 ejemplares, ICML-UNAM 12319, E63; 4 ejemplares, ICML-UNAM 12328, E64; 32 ejemplares, ICML-UNAM 12428, E76; 3 ejemplares, ICML-UNAM 12436, E76. Tabla 1.

Familia Euryalidae Gray, 1840. 12 ejemplares en una especie.

*2.- Asteroschema arenosum Lyman, 1878 (Figs. 2A, 2B).

Referencia de identificación: Lyman 1878b: 235, Lám. 3, Figs. 62-64.

Material examinado: 12 ejemplares. Los ejemplares correspondieron al área de Quintana Roo, en un rango batimétrico de 613-1,040 m, asociada a coral blando. Un ejemplar, ICML-UNAM 3.215.2, E15; 1 ejemplar, ICML-UNAM 9813, E46; 5 ejemplares, ICML-UNAM 9798, E50; 5 ejemplares, ICML-UNAM 9804, E51. Tabla 1.

Familia Gorgonocephalidae Ljungman, 1867. Diez ejemplares en dos especies.

\section{3.- Astracme mucronata (Lyman, 1869)}

Referencias de identificación: Astrophyton mucronatum Lyman, 1869: 348. Astracme mucronata en Laguarda-Figueras et al., 2009: 62, Lám. 13, se proporciona diagnosis, distribución geográfica y batimétrica.

Material examinado: Nueve ejemplares. Los ejemplares correspondieron al área de Yucatán, en un rango batimétrico de 254-351 m. Un ejemplar, ICML-UNAM 3.183.1, E28; 2 ejemplares, ICML-UNAM 3.183.2, E29; 3 ejemplares, ICML-UNAM 9634, E30; 3 ejemplares, ICML-UNAM 12306, E62. Tabla 1.

*4.- Astrogomphus rudis Verrill, 1899 (Figs. 2C, 2D).

Referencia de identificación: Verrill, 1899: 84, Lám. 7, Figs. 1, 1 A.
Material examinado: Un ejemplar, ICMLUNAM 3.165.0, E12, del área de Yucatán a 215 m. Tabla 1.

II. Orden Ophiurida Müller \& Troschel, 1840 (restringido sensu O'Hara et al., 2017)

Suborden Ophiomusina O'Hara et al., 2017 Familia Ophiosphalmidae O'Hara et al., 2018. 586 ejemplares en cinco especies.

\section{5.- Ophiosphalma armigerum (Lyman, 1878)}

Referencias de identificación: Ophiomusium armigerum Lyman, 1878a: 109, Lám. 1, Figs. 21, 22. Ophiosphalma armigerum Hernández-Herrejón et al., 2008: 113. Ophiosphalma planum Laguarda-Figueras et al., 2005: 114; Laguarda-Figueras et al., 2009: 110, Lám. 37 proporcionan diagnosis, distribución geográfica y batimétrica.

Material examinado: 344 ejemplares. 127 ejemplares correspondieron al área de Yucatán y 217 ejemplares a la de Quintana Roo, en un rango batimétrico de 359-1,040 m.

2 ejemplares, ICML-UNAM 3.130.2, E17; 2 ejemplares, ICML-UNAM 3.200.22, E25; 2 ejemplares, ICML-UNAM 9645, E31; 2 ejemplares, ICML-UNAM 9698, E38; 69 ejemplares, ICML-UNAM 9733, E42; 95 ejemplares, ICML-UNAM 9751, E43; 1 ejemplar, ICMLUNAM 9763, E44; 1 ejemplar, ICML-UNAM 9792, E49; 4 ejemplares, ICML-UNAM 9808, E51; 1 ejemplar, ICML-UNAM 12203, E52; 35 ejemplares, ICML-UNAM 12219, E54; 4 ejemplares, ICML-UNAM 12240, E56; 2 ejemplares, ICML-UNAM 12256. E57; 2 ejemplares, ICML-UNAM 12266, E58; 1 ejemplar, ICMLUNAM 12279, E59; 1 ejemplar, ICML-UNAM 12288, E60; 1 ejemplar, ICML-UNAM 12323, E63; 2 ejemplares, ICML-UNAM 12330, E64; 12 ejemplares, ICML-UNAM 12336, E65; 39 ejemplares, ICML-UNAM 12345, E66; 10 ejemplares, ICML-UNAM 12354, E67; 32 ejemplares, ICML-UNAM 12366, E68; 11 ejemplares, ICML-UNAM 12392, E70; 8 ejemplares, ICML-UNAM 12396, E71; 1 ejemplar, ICML-UNAM 12404, E72; 2 ejemplares, 


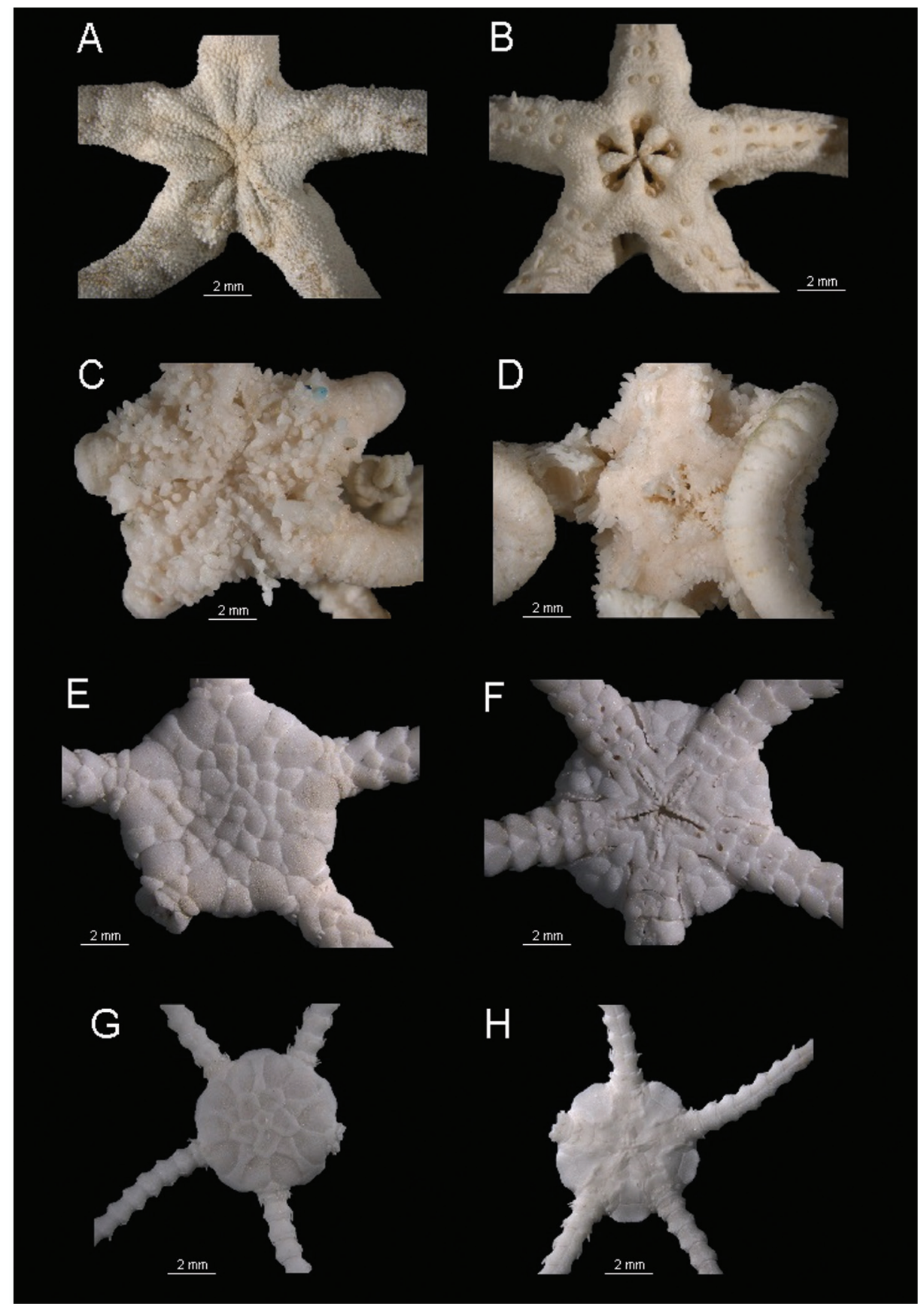

Fig. 2. Asteroschema arenosum Lyman, 1878. A. Vista aboral. B. Vista oral. Astrogomphus rudis Verrill, 1899. C. Vista aboral. D. Vista oral. Ophiosphalma monoplax (H.L. Clark, 1915). E. Vista aboral. F. Vista oral. Ophiomusa alecto (A.H. Clark, 1936). G. Vista aboral. H. Vista oral.

Fig. 2. Asteroschema arenosum Lyman, 1878. A. Aboral view. B. Oral view. Astrogomphus rudis Verrill, 1899. C. Aboral view. D. Oral view. Ophiosphalma monoplax (H.L. Clark, 1915). E. Aboral view. F. Oral view. Ophiomusa alecto (A.H. Clark, 1936). G. Aboral view. H. Oral view. 
ICML-UNAM 12413, E73; 2 ejemplares, ICML-UNAM 12438, E76. Tabla 1.

*6.- Ophiosphalma monoplax (H.L. Clark, 1915) (Figs. 2E, 2F).

Referencia de identificación: Ophiomusium monoplax H.L. Clark, 1915: 331, Lám. 20, Figs. 9, 10.

Material examinado: Dos ejemplares. Un espécimen del área de Yucatán y el otro del área de Quintana Roo obtenidos en 613 y 863 m respectivamente. Un ejemplar, ICML-UNAM 3.103.4, E15; 1 ejemplar, ICML-UNAM 3.212.3, E27. Tabla 1.

7.- Ophiosphalma serratum (Lyman, 1878)

Referencias de identificación: Ophiomusium serratum Lyman, 1878a: 109, Lám. 1, Figs. 23-25; Barker, 2016: 26, Figs. 42a-42c; Hernández-Herrejón et al., 2008: 112, Figs. 6e, 6f, presentan diagnosis, descripción y distribución geográfica y batimétrica.

Material examinado: 34 ejemplares. Correspondieron ocho ejemplares al área de Yucatán y 26 a la de Quintana Roo, en un rango batimétrico de 427-645 m. Ocho ejemplares, ICML-UNAM 3.230.0, E8; 5 ejemplares, ICML-UNAM 3.103.3, E14; 2 ejemplares, ICML-UNAM 3.130.0, E15; 2 ejemplares, ICML-UNAM 3.130.1, E17; 16 ejemplares, ICML-UNAM 3.130.6, E17; 1 ejemplar, ICMLUNAM 3.103.2. E18. Tabla 1.

\section{8.- Ophiolipus agassizii Lyman, 1878}

Referencias de identificación: Ophiolipus agassizii Lyman, 1878b: 220, Lám. 3, Figs. 52-54. Hernández-Herrejón et al., 2008: 115, Figs. 7 E, F, presentan diagnosis, descripción y distribución geográfica y batimétrica.

Material examinado: 170 ejemplares. Correspondieron 151 ejemplares al área de Yucatán y 19 a la de Quintana Roo, en un rango batimétrico de 336-650 m.

2 ejemplares, ICML-UNAM 3.200.15, E15; 2 ejemplares, ICML-UNAM 3.200.20, E23; 1 ejemplar, ICML-UNAM 3.200.21, E24; 1 ejemplar, ICML-UNAM 3.200.2, E25; 2 ejemplares, ICML-UNAM 9698, E38; 7 ejemplares, ICML-UNAM 9709b, E40; 7 ejemplares, ICML-UNAM 12278b, E54; 1 ejemplar, ICML-UNAM 12266, E58; 1 ejemplar, ICMLUNAM 12310, E62; 1 ejemplar, ICML-UNAM 12322, E63; 6 ejemplares, ICML-UNAM 12346b, E66; 24 ejemplares, ICML-UNAM 12365b, E68; 5 ejemplares, ICML-UNAM 12379, E69; 1 ejemplar, ICML-UNAM 12397b, E71; 36 ejemplares, ICML-UNAM 12426, E75; 70 ejemplares, ICML-UNAM 12427, E75; 3 ejemplares, ICML-UNAM 12439, E76. Tabla 1.

9.- Ophiomusium eburneum Lyman, 1869

Referencias de identificación: Ophiomusium eburneum Lyman, 1869: 322; 1871: Lám. 2, Figs. 1-3. Hernández-Herrejón et al., 2008: 108, Figs. 5C, 5D, presentan diagnosis, descripción, distribución geográfica y batimétrica. Benavides-Serrato et al., 2011: 357.

Material examinado: 36 ejemplares. Correspondieron cuatro ejemplares al área de Yucatán y 32 a la de Quintana Roo, en un rango batimétrico de 455-806 m. 3 ejemplares, ICML-UNAM 3.200.13, E11; 8 ejemplares, ICML-UNAM 3.200.14, E14; 3 ejemplares, ICML-UNAM 3.219.0, E15; 3 ejemplares, ICML-UNAM 3.200.15, E15; 2 ejemplares, ICML-UNAM 3.200.16, E16; 1 ejemplar, ICML-UNAM 3.200.21, E24; 2 ejemplares, ICML-UNAM 9720, E41; 2 ejemplares, ICML-UNAM 9770, E45; 5 ejemplares, ICML-UNAM 9785, E48; 7 ejemplares, ICML-UNAM 12255, E57. Tabla 1.

Familia Ophiomusaidae O'Hara et al., 2018. 618 ejemplares en tres especies.

\section{0.- Ophiomusa acufera Lyman, 1875}

Referencias de identificación: Ophiomusium acuferum Lyman, 1875: 7-8, Lám. 1, Figs. 1-5; Laguarda-Figueras et al., 2005: 114; Hernández-Herrejón et al., 2008: 106, Figs. 5a, $5 \mathrm{~b}$ (presentan diagnosis, descripción y distribución geográfica y batimétrica), BenavidesSerrato et al., 2011: 356.

Material examinado: 127 ejemplares. Correspondieron 126 ejemplares al área de 
Yucatán y uno a la de Quinta Roo, en un rango batimétrico de 214-613 m. Un ejemplar, ICMLUNAM 3.129.2, E5; 1 ejemplar, ICML-UNAM 3.129.5. E5, 107 ejemplares, ICML-UNAM 3.129.3. E6, 2 ejemplares, ICML-UNAM 3.129.6, E7; 3 ejemplares, ICML-UNAM 3.129.4, E8; 12 ejemplares, ICML-UNAM 3.129.7, E13; 1 ejemplar, ICML-UNAM 3.200.15b, E15. Tabla 1.

*11.- Ophiomusa alecto (A.H. Clark, 1936) (Figs. 2G, 2H).

Referencia: Ophiomusium alecto A. H. Clark, 1936: Lám. 2, Figs. 5, 6.

Material examinado: 24 ejemplares. Los ejemplares correspondieron 12 al área de Yucatán y 12 ejemplares a Quintana Roo, en un rango batimétrico de 214-806 m. 2 ejemplares, ICML-UNAM 3.153.3, E6; 8 ejemplares, ICML-UNAM 3.230.0, E8; 1 ejemplar, ICML-UNAM 3.153.2, E11; 1 ejemplar, ICML-UNAM 3.153.0, E13; 10 ejemplares, ICML-UNAM 3.153.1, E18; 2 ejemplares, ICML-UNAM 9770, E45. Tabla 1.

12.- Ophiomusa lymani (Wyville-Thomson, 1873)

Referencias de identificación: Ophiomusium lymani Wyville-Thomson, 1873: 172, Figs. 32-33; Lyman, 1878a: 113; HernándezHerrejón et al., 2008: 109, Figs. 6a, 6b; Laguarda-Figueras et al., 2009: 100, Lám. 32. Se presenta diagnosis, descripción y distribución geográfica y batimétrica en Hernández- Herrejón et al. (2008) y Benavides-Serrato et al., 2011: 359 .

Material examinado: 467 ejemplares. Correspondieron 141 ejemplares al área de Yucatán y 326 a la de Quinta Roo, en un rango batimétrico de 336-1 $040 \mathrm{~m} .13$ ejemplares, ICML-UNAM 3.87.4, E14; 46 ejemplares, ICML-UNAM 3.87.6, E14; 1 ejemplar, ICML-UNAM 3.87.3, E15; 1 ejemplar, ICMLUNAM 3.87.7, E15; 13 ejemplares, ICMLUNAM 3.87.9, E15; 1 ejemplar, ICML-UNAM 3.219.0, E15, 1 ejemplar, ICML-UNAM 3.87.8, E16; 8 ejemplares, ICML-UNAM 3.87.5, E17; 2 ejemplares, ICML-UNAM 3.87.10, E18,
2 ejemplares, ICML-UNAM 9699, E38; 4 ejemplares, ICML-UNAM 9701, E38; 13 ejemplares, ICML-UNAM 9709, E40; 2 ejemplares, ICML-UNAM 9721, E41; 54 ejemplares, ICML-UNAM 9734, E42; 36 ejemplares, ICML-UNAM 9752, E43; 1 ejemplar, ICMLUNAM 9762, E44; 7 ejemplares, ICML-UNAM 9816, E46; 10 ejemplares, ICML-UNAM 9806, E51; 11 ejemplares, ICML-UNAM 12202, E51; 1 ejemplar, ICML-UNAM 12208, E53; 46 ejemplares, ICML-UNAM 12218, E54; 6 ejemplares, ICML-UNAM 12294, E55; 7 ejemplares, ICML-UNAM 12238, E56; 23 ejemplares, ICML-UNAM 12253, E57; 13 ejemplares, ICML-UNAM 12254, E57; 2 ejemplares, ICML-UNAM 12278, E59; 1 ejemplar, ICML-UNAM 12287, E60; 1 ejemplar, ICML-UNAM 12303, E61; 2 ejemplares, ICML-UNAM 12311, E62; 5 ejemplares, ICML-UNAM 12337, E65; 60 ejemplares, ICML-UNAM 12346, E66; 8 ejemplares, ICML-UNAM 12355, E67; 53 ejemplares, ICML-UNAM 12365, E68; 5 ejemplares, ICML-UNAM 12391, E70; 4 ejemplares, ICML-UNAM 12397, E71; 1 ejemplar, ICML-UNAM 12405, E72; 1 ejemplar, ICMLUNAM 12414, E73; 1 ejemplar, ICML-UNAM 12440, E78. Tabla 1.

Suborden Ophiurina Müller \& Troschel, 1840 sensu O'Hara et al. (2017).

Familia Ophiuridae Müller \& Troschel, 1840 restringido sensu O'Hara et al. (2018). 604 ejemplares en seis especies.

*13.- Ophioplinthus abyssorum (Lyman, 1883) (Figs. 3A, 3B).

Referencias de identificación: Ophioglypha abyssorum Lyman, 1883: 238, Lám. 3, Figs. 25-27. Homophiura abyssorum en Paterson, 1985: 138, Fig. 52.

Material examinado: Dos ejemplares, se obtuvieron en el área de Quintana Roo, en 814 m. Un ejemplar, ICML-UNAM 9777, E47, 1 ejemplar, ICML-UNAM 9776, E47. Tabla 1.

14.- Ophiura fallax Cherbonnier, 1959 


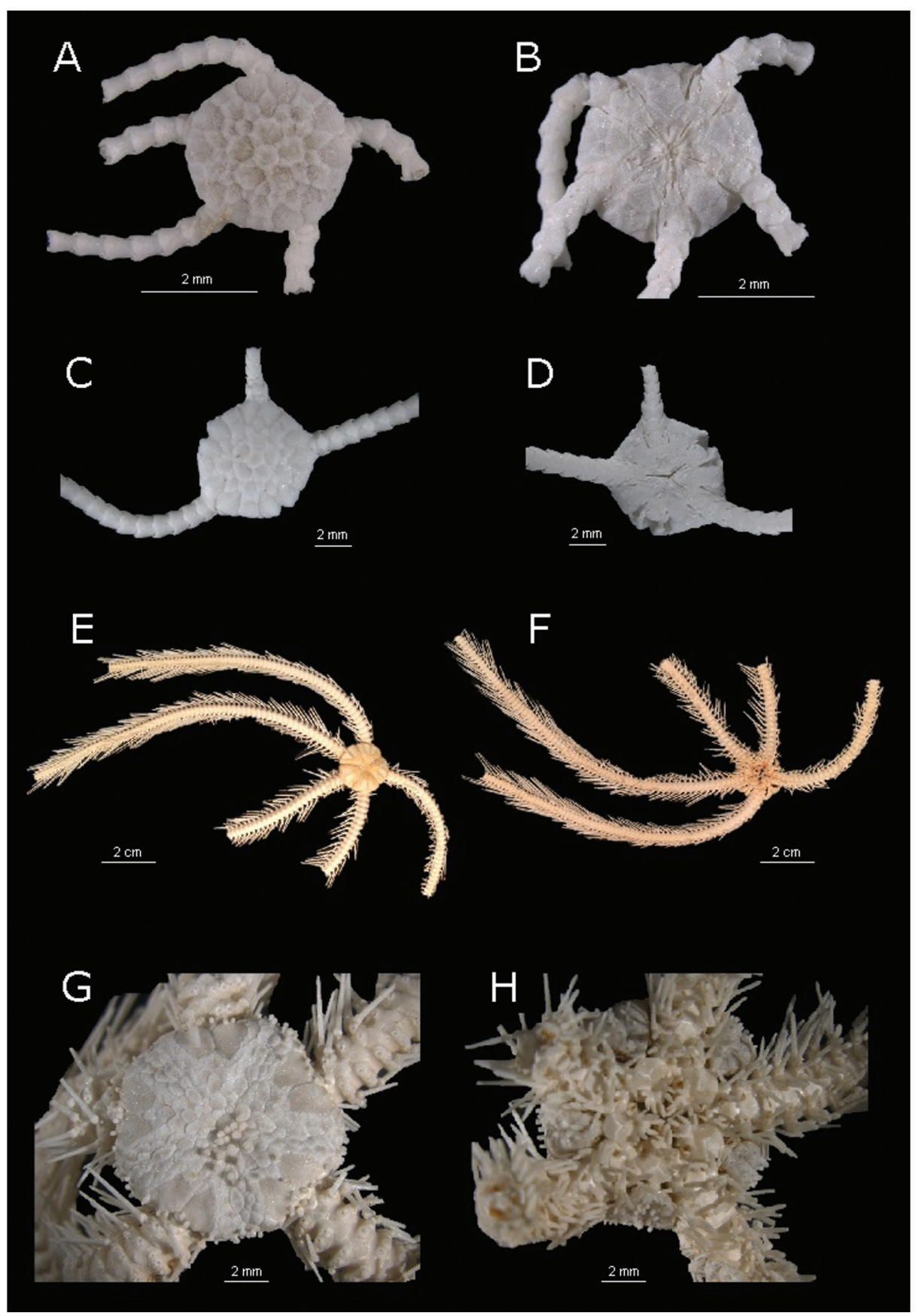

Fig. 3. Ophioplinthus abyssorum (Lyman, 1883). A. Vista aboral. B. Vista oral. Ophiuroglypha irrorata irrorata (Lyman, 1878). C. Vista aboral. D. Vista oral. Ophiocamax fasciculata Lyman, 1883. E. Vista aboral. F. Vista oral. Ophiomitra valida Lyman, 1869. G. Vista aboral. H. Vista oral.

Fig. 3. Ophioplinthus abyssorum (Lyman, 1883). A. Aboral view. B. Oral view. Ophiuroglypha irrorata irrorata (Lyman, 1878). C. Aboral view. D. Oral view. Ophiocamax fasciculata Lyman, 1883. E. Aboral view. F. Oral view. Ophiomitra valida Lyman, 1869. G. Aboral view. H. Oral view. 
Referencias de identificación: Ophiura fallax Cherbonnier, 1959: 263-265, Figs. 7a-7d., en Hernández- Herrejón et al., 2008: 98, Figs. 3 a-b., diagnosis, descripción y distribución geográfica y batimétrica.

Material examinado: 525 ejemplares. Los organismos se obtuvieron en el área de Yucatán, en un rango batimétrico de 245-315 m. 23 ejemplares, ICML-UNAM 3.124.15, E5; 448 ejemplares, ICML-UNAM 3.124.14, E6; 5 ejemplares, ICML-UNAM 3.124.16, E7; 49 ejemplares, ICML-UNAM 3.124.17, E9. Tabla 1 .

*15.- Ophiuroglypha irrorata irrorata (Lyman, 1878) (Figs. 3C, 3D).

Referencias de identificación: Ophioglypha irrorata Lyman, 1878a: 73, Lám. 4, Figs. 106-108. Ophiura (Ophiuroglypha) irrorata irrorata Paterson, 1985: 123, Figs. 46-47.

Material examinado: Cuatro ejemplares. Correspondieron tres ejemplares al área de Yucatán y uno a la de Quintana Roo, en un rango batimétrico de $394-580 \mathrm{~m}$. Un ejemplar, ICML-UNAM 3.110.2, E3; 2 ejemplares, ICML-UNAM 3.110.3, E8; 1 ejemplar, ICMLUNAM 12204, 1, E52. Tabla 1.

\section{6.- Ophiura ljungmani (Lyman, 1878)}

Referencias de identificación: Ophioglypha ljungmani Lyman, 1878a: 71-72, Lám. 3, Fig. 77; Lyman, 1882: 44, Lám. 4, Figs. 8-10. Se presenta diagnosis, descripción y distribución geográfica y batimétrica en Hernández-Herrejón et al., 2008: 101, Figs. 3e, 3 f.

Material examinado: 55 ejemplares. Organismos obtenidos en el área de Yucatán, en un rango batimétrico de $246-427 \mathrm{~m} .53$ ejemplares, ICML-UNAM 3.133.3., E5; Dos ejemplares, ICML-UNAM 3.133.2, E8. Tabla 1.

\section{7.- Ophiura sarsii (Lütken, 1855)}

Referencias de identificación: Ophioglypha sarsii Lyman, 1882: 35. diagnosis, distribución geográfica y batimétrica en Laguarda-Figueras et al., 2009: 76, Lám. 20.

Material examinado: Tres ejemplares. Organismos obtenidos en el área de Yucatán, en un rango batimétrico de 311-359 m. Un ejemplar, ICML-UNAM 3.220.0, E2; 1 ejemplar, ICML-UNAM 3.220.1. E9; 1 ejemplar, ICML-UNAM 3.220.2, E9. Tabla 1.

18.- Ophiura (Ophiura) scomba Paterson, 1985

Referencias de identificación: Ophiura scomba Paterson, 1985: 125-127, Fig. 46, Figs. 56 a-d, diagnosis, distribución geográfica y batimétrica en Laguarda-Figueras et al., 2009: 78, Lám. 21.

Material examinado: 15 ejemplares. Correspondieron 12 ejemplares al área de Yucatán y tres a la de Quintana Roo, en un rango batimétrico de 308-1,040 m. Un ejemplar, ICML-UNAM 3.223.0, E3; 1 ejemplar, ICML-UNAM 3.221.0, E17; 11 ejemplares, ICML-UNAM 9656, E32; 1 ejemplar, ICMLUNAM 9681, E38; 1 ejemplar, ICML-UNAM 9810, E51. Tabla 1.

Familia Ophiopyrgidae Perrier, 1893. 39 ejemplares en una especie.

19.- Amphiophiura metabula H.L. Clark, 1915

Referencias de identificación: Amphiophiura metabula H.L. Clark, 1915: 311-312, Lám. 17, Figs. 1-3, diagnosis, distribución geográfica y batimétrica en Laguarda-Figueras et al., 2009: 80, Lám. 22, y Benavides-Serrato et al., 2011: 268.

Material examinado: 39 ejemplares. Correspondieron seis ejemplares al área de Yucatán y 33 a la de Quintana Roo, en un rango batimétrico de 530-976 m. Cuatro ejemplares, ICML-UNAM 3.217.0, E14; 1 ejemplar, ICML-UNAM 9701, E38; 3 ejemplares, ICML-UNAM 9735, E42; 5 ejemplares, ICMLUNAM 9753, E43; 1 ejemplar, ICML-UNAM 9817, E46; 1 ejemplar, ICML-UNAM 9787, E48; 1 ejemplar, ICML-UNAM 12204, E52; 12 ejemplares, ICML-UNAM 12220, E54; 1 ejemplar, ICML-UNAM 12295, E55; 1 ejemplar, ICML-UNAM 12241, E56; 1 ejemplar, ICML-UNAM 12258, E57; 1 ejemplar, ICMLUNAM 12268, E58; 1 ejemplar, ICML-UNAM 
12280, E59; 1 ejemplar, ICML-UNAM 12289, E60; 1 ejemplar, ICML-UNAM 12348, E66; 1 ejemplar, ICML-UNAM 12369, E68; 2 ejemplares, ICML-UNAM 12415. E73, 1 ejemplar, ICML-UNAM 12441, E76. Tabla 1.

Superorden Ophintegrida O'Hara et al., 2017

III. Orden Ophiacanthida O'Hara et al., 2017

Suborden Ophiacanthina O'Hara et al., 2017 Familia Ophiocamacidae O'Hara et al., 2018. 366 ejemplares en una especie.

*20.- Ophiocamax fasciculata Lyman, 1883 (Figs. 3E, 3F).

Referencia: Ophiocamax fasciculata Lyman, 1883: 265, Lám. 7, Figs. 92-94. Benavides-Serrato et al., 2011: 350 presentan sinonimia, descripción y distribución.

Material examinado: 366 ejemplares. Correspondieron 11 ejemplares al área de Yucatán y 355 a la de Quintana Roo, en un rango batimétrico de 308-642 m. 11 ejemplares, ICML-UNAM 9653, E32; 33 ejemplares, ICML-UNAM 9717, E41; 14 ejemplares, ICML-UNAM 12242, E56; 164 ejemplares, ICML-UNAM 12243, E56; 70 ejemplares, ICML-UNAM 12244, E56; 74 ejemplares, ICML-UNAM-12245, E56. Tabla 1.

Familia Ophiotomidae Paterson, 1985 stat. nov. O’Hara et al. (2018). 134 ejemplares en tres especies.

*21.- Ophiomitra valida Lyman, 1869 (Figs. 3G, 3H).

Referencias de identificación: Lyman, 1869: 325; 1871: Lám. 2, f, Figs. 4-6; Benavides-Serrato et al., 2011: 356 presentan sinonimia, descripción y distribución.

Material examinado: 43 ejemplares, correspondientes al área de Yucatán, en un rango batimétrico de $422-426 \mathrm{~m} .8$ ejemplares, ICML-UNAM 9642, E31; 35 ejemplares, ICML-UNAM 12327, E64. Tabla 1.
*22.- Ophiotreta sertata (Lyman, 1869) (Figs. 4A, 4B).

Referencias de identificación: Ophiomitra sertata Lyman, 1869: 326; Benavides-Serrato et al., 2011: 264, presentan sinonimia, descripción y distribución.

Material examinado: 78 ejemplares. Correspondieron 70 ejemplares al área de Yucatán y ocho a la de Quintana Roo, se obtuvo en un rango batimétrico de 358 a 1 071 m. 16 ejemplares, ICML-UNAM 3.232.6, E20; 1 ejemplar, ICML-UNAM 3.232.7, E21; 2 ejemplares, ICML-UNAM 3.232.8, E22; 2 ejemplares, ICML-UNAM 9819, E46; 5 ejemplares, ICML-UNAM 12239, E56; 1 ejemplar, ICML-UNAM 12259, E57; 7 ejemplares, ICML-UNAM 12320, E63; 6 ejemplares, ICML-UNAM 12347, E66; 3 ejemplares, ICML-UNAM 12356, E67; 3 ejemplares, ICML-UNAM 12368, E68; 2 ejemplares, ICML-UNAM 12390, E70; 1 ejemplar, ICML-UNAM 12403, E72; 10 ejemplares, ICML-UNAM 12410, E73; 13 ejemplares, ICML-UNAM 12437, E76; 2 ejemplares, ICML-UNAM 12448, E77; 4 ejemplares, ICML-UNAM 12455, E78. Tabla 1.

*23.- Ophiotreta valenciennesi (Lyman, 1879) (Figs. 4C, 4D).

Referencias de identificación: Ophiacantha valenciennesi Lyman, 1879: 57, Lám. 15, Figs. 408-410; Ophiotreta valenciennesi rufescens Paterson, 1985: 49, Fig. 21; Benavides-Serrato et al., 2011: 265, presentan sinonimia, descripción y distribución.

Material examinado: 13 ejemplares. Correspondieron 12 ejemplares al área de Yucatán y uno a la de Quintana Roo, en rango batimétrico de 422-606 $\mathrm{m}$. Un ejemplar, ICML-UNAM 3.147.2, E11; 8 ejemplares, ICML-UNAM 9643, E31; 1 ejemplar, ICMLUNAM 12205, E52; 2 ejemplares, ICMLUNAM 12329, E64; 1 ejemplar, ICML-UNAM 12357, E67. Tabla 1.

Familia Ophiacanthidae Ljungman, 1867. 84 ejemplares en seis especies. 


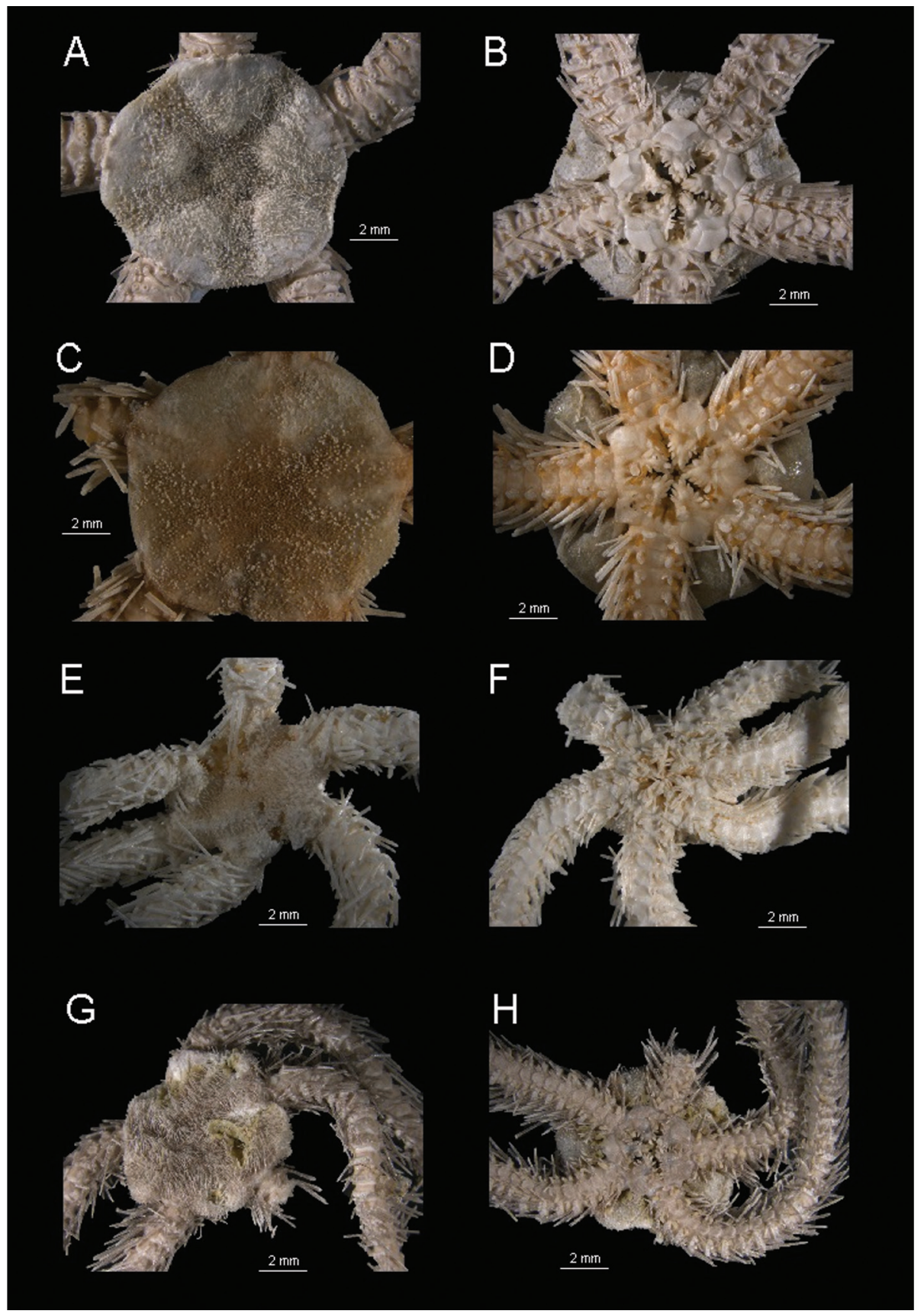

Fig. 4. Ophiotreta sertata (Lyman, 1869). A. Vista aboral. B. Vista oral. Ophiotreta valenciennesi (Lyman, 1879). C. Vista aboral. D. Vista oral. Ophiacantha anomala Sars, 1872. E. Vista aboral. F. Vista oral. Ophiacantha echinulata Lyman, 1878. G. Vista aboral. H. Vista oral.

Fig. 4. Ophiotreta sertata (Lyman, 1869). A. Aboral view. B. Oral view. Ophiotreta valenciennesi (Lyman, 1879). C. Aboral view. D. Oral view. Ophiacantha anomala Sars, 1872. E. Aboral view. F. Oral view. Ophiacantha echinulata Lyman, 1878. G. Aboral view. H. Oral view. 
*24.- Ophiacantha anomala Sars, 1872 (Figs. 4E, 4F).

Referencias de identificación: Ophiacantha anomala Sars, 1872: 12; Verrill, 1899: 36; Paterson, 1985: 24, Fig. 14.

Material examinado: Un ejemplar, obtenido en el área de Quintana Roo, en $700 \mathrm{~m}$. Un ejemplar, ICML-UNAM 3.216.0.b, E16. Tabla 1.

*25.- Ophiacantha echinulata Lyman, 1878 (Figs. 4G, 4H).

Referencia: Lyman, 1878b: 229, Lám. 1, Figs. 7-9; Benavides-Serrato et al., 2011: 246, presentan sinonimia, descripción y distribución.

Material examinado: Cuatro ejemplares, del área de Quintana Roo, en rango batimétrico de 633-820 m. Un ejemplar, ICML-UNAM 9666, E33, 1 ejemplar, ICML-UNAM 9694, E36; 1 ejemplar, ICML-UNAM 9754, E43; 1 ejemplar, ICML-UNAM 9774, E47. Tabla 1.

*26.- Ophiacantha metallacta H.L. Clark, 1915 (Figs. 5A, 5B).

Referencias de identificación: Ophiacantha metallacta H.L. Clark, 1915: 202; Paterson, 1985: 44, Fig. 19.

Material examinado: 19 ejemplares, obtenidos en el área de Quintana Roo, en rango batimétrico de 700 a $772 \mathrm{~m} .17$ ejemplares, ICML-UNAM 3.216.0, E16; 2 ejemplares, ICML-UNAM 9700, E39. Tabla 1.

*27.- Ophiochondrus convolutus Lyman, 1869 (Figs. 5C, 5D).

Referencias de identificación: Ophiochondrus convolutus Lyman, 1869: 328; 1871: Lám. 2, Fig. 7; Benavides-Serrato et al., 2011: 254, presentan sinonimia, descripción y distribución.

Material examinado: Tres ejemplares, obtenidos en el área de Yucatán, obtenidos en una profundidad de $547 \mathrm{~m} .3$ ejemplares, ICML-UNAM-12370, E68. Tabla 1.

*28.- Ophiomitrella laevipellis (Lyman, 1883) (Figs. 5E, 5F).

Referencias de identificación: Ophiacantha laevipellis Lyman, 1883: 259, Lám.
6, Figs. 82-84. Ophiomitrella laevipellis Verrill, 1899: 39, 43; Benavides-Serrato et al., 2011: 258, presentan sinonimia, descripción y distribución.

Material examinado: 30 ejemplares. Correspondieron siete ejemplares al área de Quintana Roo y 23 a la de Yucatán, en un rango batimétrico de 577-820 m. Un ejemplar, ICML-UNAM-9693, E36; 1 ejemplar, ICMLUNAM-9702, E39; 1 ejemplar, ICML-UNAM 9737, E42; 1 ejemplar, ICML-UNAM-9775, E47; 2 ejemplares, ICML-UNAM-12222, E54; 1 ejemplar, ICML-UNAM-12260, E57; 23 ejemplares, ICML-UNAM-12350, E66. Tabla 1.

* 29.- Ophioplinthaca incisa (Lyman, 1883) (Figs. 5G, 5H).

Referencia de identificación: Ophiomitra incisa Lyman, 1883: 263, Lám. 6, Figs. 89-91.

Material examinado: 27 ejemplares obtenidos en el área de Quintana Roo, en un rango de profundidad de 814 a $1040 \mathrm{~m}$. Un ejemplar, ICML-UNAM-9692, E36; 12 ejemplares, ICML-UNAM-9818, E46; 1 ejemplar, ICML-UNAM-9776, E47; 2 ejemplares, ICML-UNAM-9794, E49; 11 ejemplares, ICML-UNAM-9805, E51. Tabla 1.

Suborden Ophiodermatina Ljungman, 1867 Superfamilia Ophiodermatoida Ljungman, 1867

Familia Ophiodermatidae Ljungman, 1867. 33 ejemplares en una especie.

30.- Bathypectinura heros (Lyman, 1879)

Referencias de identificación: Pectinura heros Lyman, 1879: 48, Lám. 14, Figs. 389391. Bathypectinura heros (Lyman, 1879), Laguarda-Figueras et al., 2009: 160, Lám. 62. Benavides-Serrato et al., 2011: 336, presentan sinonimia, descripción y distribución.

Material examinado: 33 ejemplares. Correspondieron 31 ejemplares al área de Yucatán y dos a la de Quintana Roo, en un rango batimétrico de 540-642 m. Dos ejemplares, ICML-UNAM 9718, E41; 14 ejemplares, ICML-UNAM 12411, E73; 8 


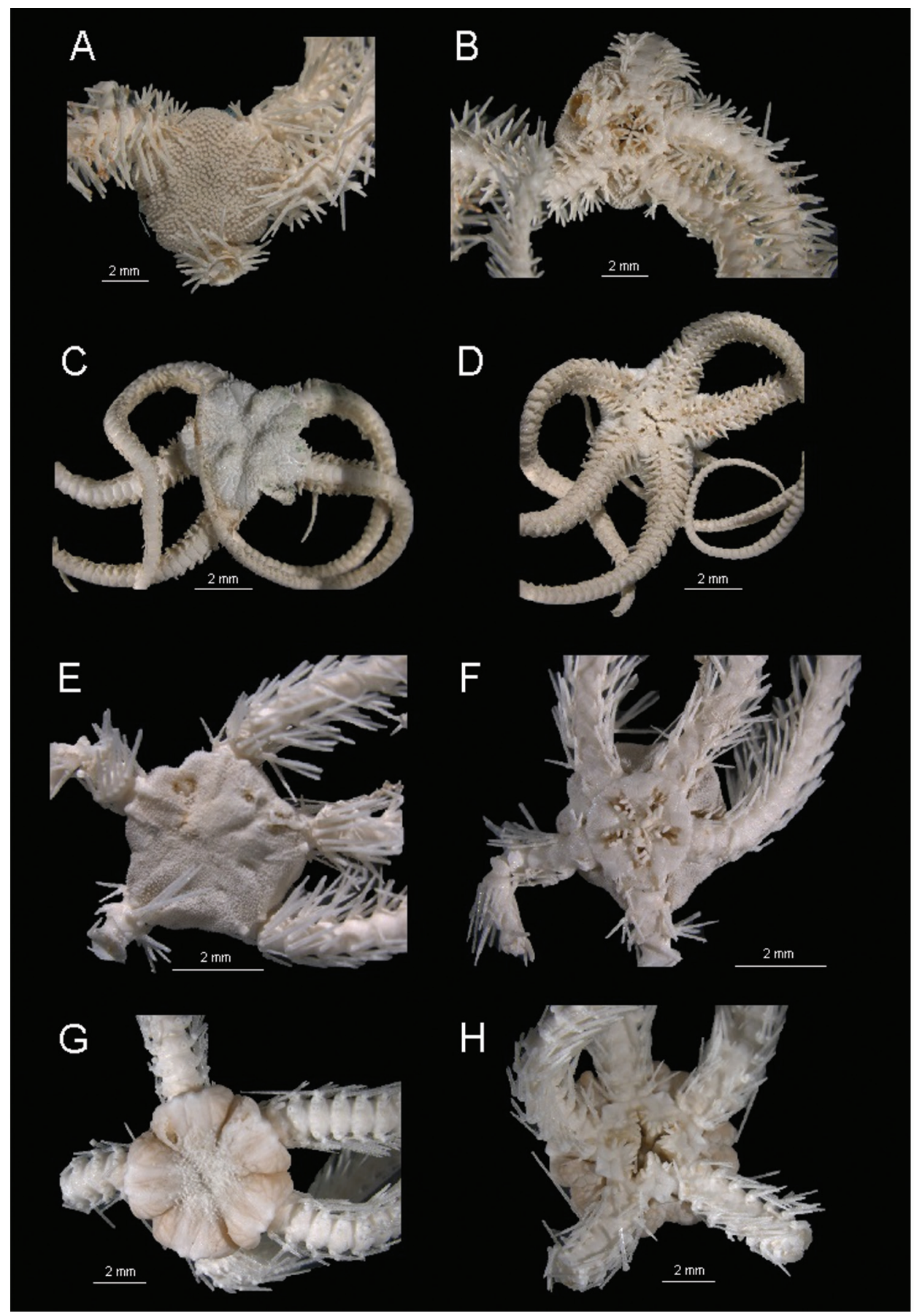

Fig. 5. Ophiacantha metallacta H.L. Clark, 1915. A. Vista aboral. B. Vista oral. Ophiochondrus convolutus Lyman, 1869. C. Vista aboral. D. Vista oral. Ophiomitrella laevipellis (Lyman, 1883). E. Vista aboral. F. Vista oral. Ohioplinthaca incisa (Lyman, 1883). G. Vista aboral. H. Vista oral.

Fig. 5. Ophiacantha metallacta H.L. Clark, 1915. A. Aboral view. B. Oral view. Ophiochondrus convolutus Lyman, 1869. C. Aboral view. D. Oral view. Ophiomitrella laevipellis (Lyman, 1883). E. Aboral view. F. Oral view. Ohioplinthaca incisa (Lyman, 1883). G. Aboral view. H. Oral view. 
ejemplares, ICML-UNAM 12412, E73; 5 ejemplares, ICML-UNAM 12435, E76; 2 ejemplares, ICML-UNAM 12447, E77; 2 ejemplares, ICML-UNAM 12454, E78. Tabla 1.

IV. Orden Ophioleucida O'Hara et al., 2017. Familia Ophiernidae O'Hara et al., 2018. 59 ejemplares en una especie.

\section{1.- Ophiernus adspersus adspersus} Lyman, 1883

Referencias de identificación: Ophiernus adspersus Lyman, 1883: 236, Lám. 3, Figs. 19-21. Ophiernus adspersus adspersus Madsen 1977: 114; Paterson, 1985: 99, Fig. 40. Diagnosis, distribución geográfica y batimétrica en Laguarda-Figueras et al., 2009: 118, Lám. 41 y Benavides-Serrato et al., 2011: 279.

Material examinado: 59 ejemplares. Correspondieron 11 ejemplares para el área de Yucatán y 48 a la de Quintana Roo, en rango batimétrico de 530 a 735 m. 4 ejemplares, ICMLUNA 9719, E41; 1 ejemplar, ICML-UNAM 9764, E44; 4 ejemplares, ICML-UNAM 9786, E48; 16 ejemplares, ICML-UNAM 12237, E56; 23 ejemplares, ICML-UNAM 12253, E57; 5 ejemplares, ICML-UNAM 12367, E68; 6 ejemplares, ICML-UNAM 12399, E71. Tabla 1.

V. Orden Amphilepidida O’Hara et al., 2017 Suborden Ophionereidina O'Hara et al.,2017 Superfamilia Ophiolepidoidea Ljungman, 1867

Familia Ophiolepididae Ljungman, 1867 (restringido). Dos ejemplares en una especie.

*32.- Ophiomastus secundus Lyman, 1878 (Figs. 6A, 6B).

Referencias de identificación: Ophiomastus secundus Lyman, 1878b: 218, Lám. 2, Figs. 16-18; Benavides-Serrato et al., 2011: 278, presentan sinonimia, descripción y distribución.

Material examinado: Dos ejemplares del área de Quintana Roo, en profundidad de 814 m. 2 ejemplares, ICML-UNAM-9773, E47. Tabla 1.
Familia Hemieuryalidae Verrill, 1899. 25

ejemplares en dos especies.

*33.- Ophiozonella marmorea (Lyman, 1883) (Figs. 5C, 5D).

Referencia: Ophizona marmorea Lyman, 1883: 233, Lám. 3, Figs. 10-12.

Material examinado: Cinco ejemplares, obtenidos en el área de Quintana Roo, en 577 m. Cinco ejemplares, ICML-UNAM 12257, E57. Tabla 1.

\section{4.- Ophiozonella nivea (Lyman, 1875)}

Referencias de identificación: Ophiozona nivea Lyman, 1875: 9, Figs. 85-86. Ophiozonella nivea Laguarda-Figueras et al., 2009: 114, Lám. 39, presentan diagnosis, distribución geográfica y batimétrica.

Material examinado: 20 ejemplares. Correspondieron 18 ejemplares al área de Yucatán y dos a la de Quintana Roo, en un rango batimétrico de 254 a $580 \mathrm{~m} .3$ ejemplares, ICML-UNAM 3.161.2, E28; 1 ejemplar, ICML-UNAM 3.161.3, E29; 1 ejemplar, ICMLUNAM 9635, E30; 1 ejemplar, ICML-UNAM 9644, E31; 1 ejemplar, ICML-UNAM 3.161.1, E31; 2 ejemplares, ICML-UNAM 9654, E32; 2 ejemplares, ICML-UNAM 12233, E52; 5 ejemplares, ICML-UNAM 12309, E62; 2 ejemplares, ICML-UNAM 12321, E63; 2 ejemplares, ICML-UNAM 12380, E69. Tabla 1.

\section{Superfamilia Ophionereidoidea Ljungman, 1867}

Familia Amphilimnidae O'Hara et al., 2018. 13 ejemplares en una especie.

\section{5.- Amphilimna olivacea (Lyman, 1869)}

Referencias de identificación: Ophiocnida olivacea Lyman, 1869: 340-341; 1871: Lám. 1, Figs. 7, 8. Amphilimna olivacea LaguardaFigueras et al., 2009: 208, Lám 86, presentan diagnosis, distribución geográfica y batimétrica y Benavides-Serrato et al., 2011: 285.

Material examinado: 13 ejemplares. Correspondieron al área de Yucatán, obtenidos en 308 y 311 m. 10 ejemplares, 


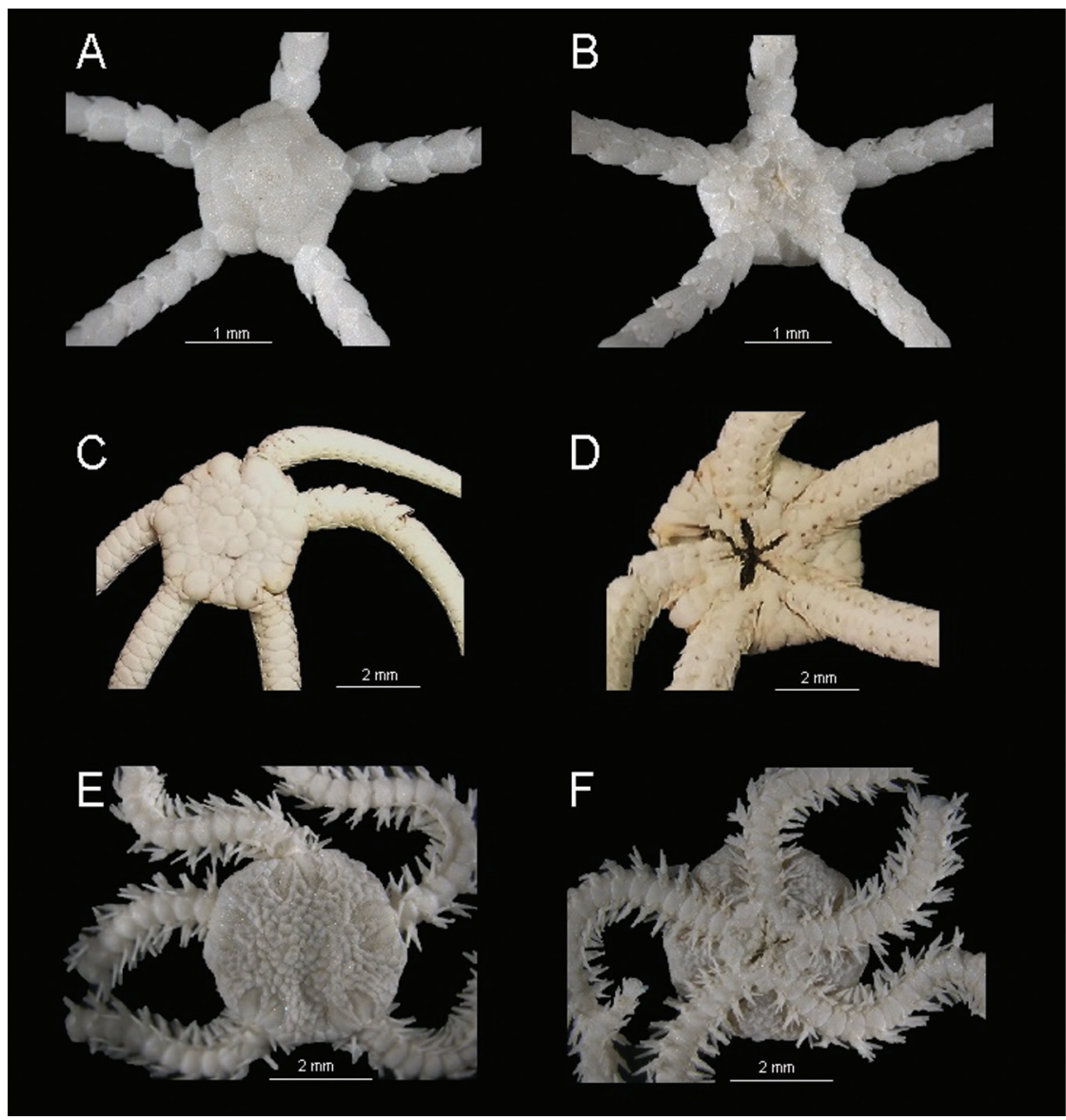

Fig. 6. Ophiomastus secundus Lyman, 1878. A. Vista aboral. B. Vista oral. Ophiozonella marmorea (Lyman, 1883). C. Vista aboral. D. Vista oral. Histampica duplicata (Lyman, 1875). E. Vista aboral. F. Vista oral.

Fig. 6. Ophiomastus secundus Lyman, 1878. A. Aboral view. B. Oral view. Ophiozonella marmorea (Lyman, 1883). C. Aboral view. D. Oral view. Histampica duplicata (Lyman, 1875). E. Aboral view. F. Oral view.

ICML-UNAM-3.119.3, E9; 3 ejemplares, ICML-UNAM-9655. E32. Tabla 1.

Familia Ophionereididae Ljungman, 1867. Seis ejemplares en una especie.

36.- Ophiochiton ternispinus Lyman, 1883

Referencias de identificación: Ophiochiton ternispinus Lyman, 1883: 255, Lám. 5, Figs. 67-69, diagnosis, distribución geográfica y batimétrica en Laguarda-Figueras et al., 2009: 144, Lám. 54 y Benavides-Serrato et al., 2011: 331.

Material examinado: Seis ejemplares. Correspondieron al área de Quintana Roo, con distribución batimétrica de 595 a 645 m. Un ejemplar, ICML-UNAM-3.203.4, E18; 1 ejemplar, ICML-UNAM- 9736, E42; 1 ejemplar, ICML-UNAM-9756, E43; 1 ejemplar, ICML-UNAM-12223, E54; 1 ejemplar, ICML-UNAM-12269, E58; 1 ejemplar, ICMLUNAM-12281, E59. Tabla 1.

Suborden Gnathophiurina Matsumoto, 1915 Superfamilia Amphiuroidea Ljungman, 1867 Familia Amphiuridae Ljungman, 1867. 39 ejemplares en seis especies.

37.- Amphioplus coniortodes H.L. Clark, 1918 
Referencias de identificación: Amphioplus coniortodes H.L. Clark, 1918: 291, Lám. 7, Figs. 3, 4. Amphioplus (Amphioplus) coniortodes Laguarda-Figueras et al., 2005, en Laguarda-Figueras et al., 2009: 192, Lám. 78, presentan diagnosis, distribución geográfica y batimétrica.

Material examinado: Dos ejemplares, obtenidos en el área de Yucatán, en 246 y $311 \mathrm{~m}$. Un ejemplar, ICML-UNAM 3.103.6, E1; 1 ejemplar, ICML-UNAM 3.103.8, E5. Tabla 1.

38.- Amphipholis januarii Ljungman, 1866

Referencias de identificación: Amphipholis januarii Ljungman, 1866: 165; LaguardaFigueras et al., 2009: 198, Lám. 81. Se presenta diagnosis, distribución geográfica y batimétrica en Hernández-Herrejón et al., 2008: 146, Figs.15c, 15d.

Material examinado: 20 ejemplares, obtenidos en el área de Yucatán, a 311 m de profundidad. 20 ejemplares, ICML-UNAM-3.190.2, E9. Tabla 1.

39.- Amphipholis squamata (Delle Chiaje, 1828)

Referencias de identificación: Amphipholis squamata (Delle Chiaje, 1828); Verrill, 1899: 24; Hernández-Herrejón et al., 2008: 146, Figs. 15e, 15f, presentan diagnosis, distribución geográfica y batimétrica y BenavidesSerrato et al., 2011: 290.

Material examinado: Cuatro ejemplares, obtenidos en el área de Quintana Roo, a 976 m de profundidad; 4 ejemplares, ICML-UNAM 9815, E46. Tabla 1.

\section{0.- Amphiura fibulata Koehler, 1914}

Referencias de identificación: Amphiura fibulata Koehler, 1914: 56, Lám. 7, Figs. 3-5. Laguarda-Figueras et al., 2005: 115; Hernández-Herrejón et al., 2008: 147, Figs. 16a, 16b; Laguarda-Figueras et al., 2009: 202, Lám. 83, presentan diagnosis, distribución geográfica y batimétrica.

Material examinado: Dos ejemplares, obtenidos en el área de Yucatán, a 311 y 427 $\mathrm{m}$ de profundidad. Un ejemplar, ICML-UNAM
3.136.2, E8; 1 ejemplar, ICML-UNAM 3.136.3, E9. Tabla 1.

\section{1.- Amphiura palmeri Lyman, 1882}

Referencias de identificación: Amphiura palmeri Lyman, 1882: 143; Laguarda-Figueras et al., 2009: 204, Lám. 84. Se presenta diagnosis, distribución geográfica y batimétrica en Hernández-Herrejón et al., 2008: 149, Figs. $16 \mathrm{c}, 16 \mathrm{~d}$.

Material examinado: Cuatro ejemplares, obtenidos en el área de Yucatán, a 311 y 315 $\mathrm{m}$ de profundidad. Un ejemplar, ICML-UNAM 3.137.2, E7; 3 ejemplares, ICML-UNAM 3.137.1, E9. Tabla 1.

\section{2.- Amphiura stimpsonii Lütken, 1859}

Referencias de identificación: Amphiura stimpsonii Lütken, 1859; Laguarda-Figueras et al., 2009: 206, Lám. 85. Se presenta diagnosis, distribución geográfica y batimétrica en Hernández-Herrejón et al., 2008: 150, Figs. $16 \mathrm{e}, 16 \mathrm{f}$.

Material examinado: Siete ejemplares, obtenidos en el área de Yucatán, en 308 y 352 m. Seis ejemplares, ICML-UNAM 3.188.2, E10; 1 ejemplar, ICML-UNAM 9657, E32. Tabla 1.

Superfamilia Ophiactoidea Ljungman, 1867. Familia Ophiothamnidae O'Hara et al., 2018. 53 ejemplares en una especie.

*43.- Histampica duplicata (Lyman, 1875) (Figs. 5E, 5F).

Referencias de identificación: Amphiura duplicata Lyman, 1875: 19, Lám. 5, Figs. 78, 87. Histampica duplicata Paterson, 1985: 80, Fig. 32; Benavides-Serrato et al., 2011: 313, presentan sinonimia, descripción y distribución.

Material examinado: 53 ejemplares, correspondieron 18 ejemplares al área de Yucatán y 35 a la de Quintana Roo, en un rango batimétrico de 426-1040 m. 2 ejemplares, ICML-UNAM 9695, E37; 1 ejemplar, ICMLUNAM 9757, E43; 12 ejemplares, ICMLUNAM 9814, E46; 1 ejemplar, ICML-UNAM 9793, E49; 2 ejemplares, ICML-UNAM 9799, 
E50; 9 ejemplares, ICML-UNAM 9807, E51; 2 ejemplares, ICML-UNAM 12221, E54; 3 ejemplares, ICML-UNAM 12267, E58; 3 ejemplares, ICML-UNAM 12282, E59; 2 ejemplares, ICML-UNAM 12331, E64; 1 ejemplar, ICML-UNAM 12349, E66; 5 ejemplares, ICML-UNAM 12398, E71; 1 ejemplar, ICMLUNAM 12419, E74; 4 ejemplares, ICMLUNAM 12429, E75; 1 ejemplar, ICML-UNAM 12438, E76; 1 ejemplar, ICML-UNAM 12440, E78. Tabla 1.

\section{DISCUSIÓN Y CONCLUSIONES}

Con el fin de valorar los esfuerzos institucionales en el desarrollo de campañas oceanográficas y mantenimiento de ejemplares en colecciones, el presente trabajo contribuye al conocimiento de la diversidad béntica marina en el Talud de la Península de Yucatán, México, generando el inventario de las especies registradas en la Colección Nacional de Equinodermos del ICML-UNAM, presentando 19 nuevos registros que han incrementado su Catálogo, ubicándolas en el nuevo contexto de la clasificación actual.

Las 43 especies fueron evaluadas en su distribución en base al número de estaciones en que estuvieron presentes y su abundancia por el número de ejemplares. Las especies de amplia distribución y abundancia son Ophiosphalma armigerum (24 estaciones), Ophiomusa lymani (28 estaciones), Ophiolipus agassizii, Ophiotreta sertata e Histampica duplicata (cada una en 16 estaciones).

En el Estado de Quintana Roo, las estaciones E15, E43, E46, E54, E56 y E57 son las de mayor diversidad, se ubican al Norte de Cabo Catoche con un rango de profundidad de 577 a 976 m, su diversidad está conformada por 18 especies: Asteroschema arenosum (dos ejemplares), Ophiosphalma armigerum (136 ejemplares), Ophiosphalma monoplax (un ejemplar), Ophiosphalma serratum (dos ejemplares), Ophiolipus agassizii (dos ejemplares), Ophiomusium eburneum (tres ejemplares), Ophiomusa lymani (148 ejemplares), Amphiophura metabula (20 ejemplares), Ophiocamax fasciculata (222 ejemplares), Ophiotreta sertata (ocho ejemplares), Ophiacantha echinulata (un ejemplar), Ophiomitrella laevipellis (tres ejemplares), Ophioplinthaca incisa (12 ejemplares), Bathypectinura heros (dos ejemplares), Ophiernus adspersus adspersus (39 ejemplares), Ophiochiton ternispinus (dos ejemplares), Amphipholis squamata (cuatro ejemplares), Histampica duplicata (15 ejemplares). Este resultado es similar al presentado por BorreroPérez, Benavides-Serrato, Solano, \& Navas (2008) y Benavides-Serrato, Borrero-Pérez, \& Díaz-Sanchez (2011) para especies obtenidas en profundidades mayores a los $200 \mathrm{~m}$ en el Caribe Colombiano, donde 19 taxa son compartidos con el presente trabajo.

En el estado de Yucatán las estaciones E66, E68 y E76 son las de mayor diversidad, se ubican al Norte de Progreso con un rango de profundidad de 547 a $611 \mathrm{~m}$, su diversidad está constituida por once especies: Asteronyx loveni (35 ejemplares), Ophiosphalma armigerum (73 ejemplares), Ophiolipus agassizii (33 ejemplares), Ophiomusa lymani (113 ejemplares), Amphiophura metabula (tres ejemplares), Ophiotreta sertata (22 ejemplares), Ophiochondrus convolutus (tres ejemplares), Ophiomitrella laevipellis (23 ejemplares), Bathypectinura heros (cinco ejemplares), Ophiernus adspersus adspersus (cinco ejemplares), e Histampica duplicata (dos ejemplares). Así mismo con menor profundidad la estación E8 (427 m) adquiere importancia para adicionar Ophiomusa serratum (ocho ejemplares), Ophiomusa acufera (tres ejemplares), Ophiomusa alecto (8 ejemplares), Ophiuroglypha irrorata irrorata (dos ejemplares), Ophiura ljungmani (dos ejemplares) y Amphiura fibulata (un ejemplar) y la estación E6 $(245 \mathrm{~m})$ con Ophiomusa acufera (107 ejemplares) y Ophiura fallax (448 ejemplares).

Con los datos específicos para cada una de las 43 especies en su distribución batimétrica los siguientes cinco registros se modifican: Ophiosphalma monoplax, Lyman, (1878) la reporta a $825 \mathrm{~m}$ y se obtuvo en 613 y $863 \mathrm{~m}$; Ophiolipus agassizii, Lyman (1882) la registra de 146 a $310 \mathrm{~m}$ y se obtuvo en 336 a $650 \mathrm{~m}$; 
Ophioplinthus abyssorum reportada a $2007 \mathrm{~m}$ por Lyman (1883) se obtuvo a $814 \mathrm{~m}$ de profundidad; Ophiura (Ophiura) scomba reportada de 358 a $3943 \mathrm{~m}$ por Paterson (1985) se obtuvo en un rango batimétrico de 308 a 1040 m; Ophiocamax fasciculata reportada de 324 a $540 \mathrm{~m}$ por Lyman (1883) se obtuvo en rango batimétrico de 308 a $642 \mathrm{~m}$.

Con el ordenamiento y seguimiento de cada uno de los registros de las diferentes especies, se ha ordenado y actualizado la información que la CNE resguarda, se confirma que son especies de aguas tropicales profundas, integrantes de uno de los biomas con la biodiversidad mas alta en ofiuroideos actualmente evaluado por O'Hara, Hugall, Woolley, Bribiesca-Contreras, \& Bax (2019). Las colecciones científicas proveen una información esencial para el entendimiento de la composición y distribución de la biodiversidad.

La biodiversidad del Talud de la zona noreste de la península de Yucatán, México está conformada por 43 especies, de las cuales 19 son nuevos registros para el área de estudio.

Declaración de ética: los autores declaran que todos están de acuerdo con esta publicación y que han hecho aportes que justifican su autoría; que no hay conflicto de interés de ningún tipo; y que han cumplido con todos los requisitos y procedimientos éticos y legales pertinentes. Todas las fuentes de financiamiento se detallan plena y claramente en la sección de agradecimientos. El respectivo documento legal firmado se encuentra en los archivos de la revista.

\section{AGRADECIMIENTOS}

Los autores agradecen a Timothy O'Hara del Museo Victoria, Australia, por su apoyo en la corroboración de las especies; a María de los Ángeles Álvarez Espíndola (ENP 4, UNAM), a Susana Guzmán Gómez técnico académico del Laboratorio de Biodiversidad del IB, UNAM por el apoyo en la toma de fotografías, y a Carlos A. Conejeros Vargas por su ayuda en la realización de las figuras del presente trabajo. Al personal del Laboratorio de Ecología Pesquera de Crustáceos del ICML, UNAM por el apoyo recibido durante las campañas oceanográficas realizadas. Al Proyecto IN223109-3 apoyado por Proyectos de Investigación e Innovación Tecnológica (PAPIIT) de la Universidad Nacional Autónoma de México.

\section{RESUMEN}

Introducción: La clase Ophiuroidea es la más diversa del Phylum Echinodermata, con 2064 especies descritas, que se pueden encontrar desde zonas intermareales hasta profundidades abisales, pero la fauna de la península de Yucatán necesita más estudio. Objetivo: Describir la diversidad de ofiuroideos en el talud continental de Yucatán. Métodos: Se recopiló información de cuatro estudios oceanográficos llevados a cabo en agosto de 2005, junio de 2007 (BIOREPES 1 y 2: BRP1 y BRP2), abril de 2011 y agosto de 2014 (COBERPES 2 y 6: CBP2 y CBP6). Los especímenes se obtuvieron mediante la pesca de arrastre con una red camaronera en sustratos blandos y con una draga de arrastre tipo skimmer en un rango de profundidad de 200 a $1071 \mathrm{~m}$ frente a los estados de Yucatán y Quintana Roo, México. Resultados: Encontramos miembros de dos superórdenes, cinco órdenes, seis subórdenes, cinco superfamilias, 18 familias, 28 géneros y 43 especies. Conclusiones: La zona noreste de la Península de Yucatán tiene al menos 43 especies, de las cuales 19 son nuevos registros para el área.

Palabras clave: taxonomía; nuevos registros; mar profundo; Golfo de México.

\section{REFERENCIAS}

Barker, A.N. (2016). As illustrated catalogue of type specimens of the bathyal brittlestar genera Ophiomusium Lyman and Ophiosphalma H.L. Clark (Echinodermata: Ophiuroidea). Zootaxa, 4097(1), 1-40.

Benavides-Serrato, M., Borrero-Pérez, G.H., \& Díaz-Sanchez, C.M. (2011). Equinodermos del Caribe colombiano I: Crinoidea, Asteroidea y Ophiuroidea. Serie de Publicaciones Especiales de Invermar, 22, 384.

Borrero-Pérez, G.H., Benavides-Serrato, M., Solano, O., \& Navas, S.G.R. (2008). Brittle-stars (Echinodermata: Ophiuroidea) from the continental shelf and upper slope of Colombian Caribbean. Revista de Biología Tropical, 56(3), 169-204.

Cherbonnier, G. (1959). Echinoderms de la Guyane francaise (Crinoides, Astérides, Ophiurides, Echinides, Holothurides). Bulletin du Museum National d'Histoire Naturelle, Series, 31(3), 261-265. 
Clark, A.H. (1936). Four new brittlestars from Puerto Rico. Reports on the collections obtained by the first Johnson Smithsonian deep-sea expedition to the Puerto Rican deep. Smithsonian Miscellaneous Collections, 91(24), 1-8.

Clark, H.L. (1915). Catalogue of Recent Ophiurans. Memoirs of the Museum of Comparative Zoology, 25(4), 163-376.

Clark, H.L. (1918). Brittle-Stars, New and Old. Bulletin Museum of Comparative Zoology, 62(6), 265-338.

Delle Chiaje, S. (1828). Memorie sulla storia e notomia degli animali senza vertebre del regno di Napoli. Società Tipografica, 3, 1-232.

Durán-González, A., Laguarda-Figueras A., Solís-Marín F.A., Buitrón-Sánchez B.E., Gust-Ahearn C. \& Torres-Vega J. (2005). Equinodermos (Equinodermata de las aguas mexicanas del Golfo de México. Revista de Biología Tropical 53(3): 53-68.

Felder, D.L., Camp D.K. \& Tunnell J.W.Jr. (2009). An Introduction to Gulf of Mexico Biodiversity Assessment. 1-13. In: Felder D.L. y Camp D.K. (Eds.) Gulf of Mexico Origin, Waters, and Biota. Vol. 1 Biodiversity, Texas A\&M University Press, U.S.A.

Hernández-Herrejón, L.A., Solís-Marín, F.A., \& LaguardaFigueras, A. (2008). Ofiuroideos (Echinodermata: Ophiuroidea) de las aguas mexicanas del golfo de México. Revista de Biología Tropical, 56(3), 83-167.

Koehler, R. (1914). A contribution to the study of Ophiurans of the United States National Museum. Smithsonian Institution. United States National Museum. Washington. Bulletin, 84: 1-173.

Laguarda-Figueras, A., Solís-Marín, F.A., Durán-González, A., Gust-Ahearn, C., Buitrón-Sánchez, B.E., \& Torres-Vega, J. (2005). Equinodermos (echinodermata) del Caribe Mexicano. Revista de Biología Tropical, 53(3), 109-122.

Laguarda-Figueras, A., Hernández-Herrejón, L.A., SolísMarín, F.A., \& Durán González, A. (2009). Ofiuroideos del Caribe Mexicano y Golfo de México. Ciudad de México: ICMyL-UNAM, CONABIO, Jiménez Editores e Impresores. 249 p.

Ljungman, A. (1867). Om nagra arter of Ophiurider. Ofversigt af Kongl. Vetenskaps Akademiens Förhandlingar, 163-166.

Lütken, C.F. (1855) Bidrag til kundstab om Slangestjernerne I. Forelöbig oversight over Grönlandshavets Ophiurer. Videnskabelige Meddelelser fra den naturhistoriske Forening i Kjöbenhavn, 1854: 95-105.

Lütken, C.F. (1859). Additamenta ad historiam Ophiuridarum. Anden Afdelning. Det kongelige danske Videnskabernes Selskabs Skrifter. 5 Raekke, Naturvidenskabelig og mathematisk Afdelning, 5, 177-271
Lyman, T. (1869). A preliminary report on the Ophiuridae and Astrophytidae dredged in deep water between Cuba and Florida Reef. Bulletin of the Museum of Comparative Zoology, 1, 309-354.

Lyman, T. (1871). Supplement to the Ophiuridae and Astrophytidae. Illustrated Catalogue of the Museum of Comparative Zoology at Harvard College, 6: 1-15.

Lyman, T. (1875). Zoölogical Results of the Hassler Expedition. II. Ophiuridae and Astrophytidae, including those dredged by the late Dr. Williams Stimpson. Illustrated Catalogue of the Museum of Comparative Zoology at Harvard College, 8, 1-34.

Lyman, T. (1878a). Ophiuridae and Astrophytidae of the exploring voyage of the H. M. S. "Challenger", under Prof. Sir Wyville Thomson, F. R. S. Part I. Bulletin of the Museum of Comparative Zoölogy, Harvard College, 5(7), 65-167.

Lyman, T. (1878b). Reports on the Results of Dredging, under the Supervision of Alexander Agassiz, in the Gulf of Mexico, by the United States Coast Survey Steamer "Blake", Lieutenant-Commander C. D. SIGSBEE, U. S. N., Commanding. II. Bulletin of the Museum of Comparative Zoölogy, Harvard College, 5(9), 181-238.

Lyman, T. (1879). Ophiuridae and Astrophytidae of the exploring voyage of the H. M. S. "Challenger", under Prof. Sir Wyville Thomson, F. R. S. Part II. Bulletin of the Museum of Comparative Zoölogy, Harvard College, 6(2), 17-83.

Lyman, T. (1882). Report on Ophiuroidea dredged by H.M.S. "Challenger" during the years 1873-1876. Report on the Scientific Results of the Voyage of H.M.S. "Challenger" during the years 1873-1876. Zoology, 5(14), 1-386.

Lyman, T. (1883). XX. Report on the Ophiuroidea. Reports on the results of dredging, under the supervision of Alexander Agassiz, in the Caribbean Sea (1878-79), and on the east coast of the United States, during the summer of 1880 , by the U. S. Coast survey Steamer "Blake", commander J. R. Bartlett, U.S.N. Commanding. Bulletin of the Museum of Comparative Zoölogy, Harvard College, 10(6), 227-287.

Madsen, F.J. (1977). The Ophioleucidae (Ophiuroidea). Galathea Report, 14, 109-122.

Matsumoto, H. (1913). Evolutionary history of the class Ophiuroidea and a note on the new classification of the class. Zoological Magazine, 25: 521-527.

Matsumoto, H. (1915). A new classification of Ophiuroidea: with description of new genera and species. Proceedings of the Academy of Natural Sciences of Philadelphia, 67: 43-92. 
Müller, J. \& Troschell, F.H. (1842). System der Asteriden. Mit Swölf Kupfertafeln, Frriedrich Vieweg und Sohn. Braunschweig, Germany, 134 p.

O’Hara, T.D., Hugall, A.F., Thuy, B. Stöhr, S., \& Martynov, A.V. (2017). Restructuring higher taxonomy using broad-scale phylogenomics: The living Ophiuroidea. Molecular Phylogenetics and Evolution, 107, 415-430.

O’Hara, T.D., Stöhr, S., Hugall, A.F., Thuy, B., \& Martynov, A. (2018). Morphological diagnoses of higher taxa in Ophiuroidea (Echinodermata) in support of a new classification. European Journal of Taxonomy, $416,1-35$.

O’Hara, T., Hugall, A., Woolley, S., Bribiesca-Contreras, G. \& Bax, N. (2019). Contrastring processes drive ophiuroid phylodiversity across shallow and deep seafloors. Nature, 565, 636-639.

Paterson, G.L.J. (1985). The Deep-sea Ophiuroidea of the North Atlantic Ocean. Bulletin of the British Museum Natural History, 49(1), 1-160.

Pawson, D. (2007). Phylum Echinodermata. Zootaxa, $1668,749-764$.

Pawson, D.L., Vance, D.J., Messing, C.G., Solís-Marín, F.A., \& Mah, C.L. (2009). Echinodermata of the Gulf of Mexico: 1177-1204. En: D.L. Feder \& D. K. Camp (Eds.), Gulf of Mexico. Origin, Waters, and Biota pp. 1177-1204). USA: Texas A\&M University Press.
Sars, G.O. (1872). Nye Echinodermer fra den norske kyst. Forhandlinger $i$ Videnskabs-selskabet $i$ Christiania. 1871: 1-31.

Stöhr, S., O’Hara, T.D., \& Thuy, B. (2012). Global Diversity of Brittle Stars (Echinodermata: Ophiuroidea). PLoS One, 7(3), e9140.

Stöhr, S., O’Hara, T., \& Thuy, B. (2020). World Ophiuroidea Database. Ophiomusina. Accessed through: World Register of Marine Species at: http://www.marinespecies.org/aphia.php? $\mathrm{p}=$ taxdetails $\& i d=1075310$ on 2020-03-12.

Solís-Marín, F. A., Laguarda-Figueras, A. y Honey-Escandón, M. (2014). Biodiversidad de equinodermos (Echinodermata) en México. Revista Mexicana de Biodiversidad, Supl. 85: S441-S449.

Verrill, A.E. (1899). Report on the Ophiuroidea collected by the Bahama Expedition from the University of Iowa in 1893. Bulletin from the Laboratories of Natural History of the State University of Iowa, 5(7), 1-86.

Woolley, S.N.C., Tittensor, D.P., Dunstan, P.K., GuilleraArroita, G., Lahoz-Monfort, J.J.L. Wintle, B.A., Worm, B., \& O'Hara, T.D. (2016). Deep-sea diversity patterns are shaped by energy availabity. Nature, 17937.

Wyville-Thomson, C. (1873). The Depths of the Sea. Macmillan \& Co. London. 527 pp. 